\title{
Approximation of complex algebraic numbers by algebraic numbers of bounded degree
}

\author{
YANN BUGEAUD AND JAN-HENDRIK EVERTSE
}

\begin{abstract}
To measure how well a given complex number $\xi$ can be approximated by algebraic numbers of degree at most $n$ one may use the quantities $w_{n}(\xi)$ and $w_{n}^{*}(\xi)$ introduced by Mahler and Koksma, respectively. The values of $w_{n}(\xi)$ and $w_{n}^{*}(\xi)$ have been computed for real algebraic numbers $\xi$, but up to now not for complex, non-real algebraic numbers $\xi$. In this paper we compute $w_{n}(\xi), w_{n}^{*}(\xi)$ for all positive integers $n$ and algebraic numbers $\xi \in \mathbf{C} \backslash \mathbf{R}$, except for those pairs $(n, \xi)$ such that $n$ is even, $n \geq 6$ and $n+3 \leq \operatorname{deg} \xi \leq 2 n-2$. It is known that every real algebraic number of degree $>n$ has the same values for $w_{n}$ and $w_{n}^{*}$ as almost every real number. Our results imply that for every positive even integer $n$ there are complex algebraic numbers $\xi$ of degree $>n$ which are unusually well approximable by algebraic numbers of degree at most $n$, i.e., have larger values for $w_{n}$ and $w_{n}^{*}$ than almost all complex numbers. We consider also the approximation of complex non-real algebraic numbers $\xi$ by algebraic integers, and show that if $\xi$ is unusually well approximable by algebraic numbers of degree at most $n$ then it is unusually badly approximable by algebraic integers of degree at most $n+1$. By means of Schmidt's Subspace Theorem we reduce the approximation problem to compute $w_{n}(\xi), w_{n}^{*}(\xi)$ to an algebraic problem which is trivial if $\xi$ is real but much harder if $\xi$ is not real. We give a partial solution to this problem.
\end{abstract}

Mathematics Subject Classification (2000): 11J68.

\section{Introduction}

Conjecturally, most of the properties shared by almost all numbers (throughout the present paper, 'almost all' always refers to the Lebesgue measure) should be either trivially false for the algebraic numbers, or satisfied by the algebraic numbers. Thus, the sequence of partial quotients of every real, irrational algebraic number of degree at least 3 is expected to be unbounded, and the digit 2 should occur infinitely often in the decimal expansion of every real, irrational algebraic number. Our very limited knowledge on these two problems show that they are far from being solved.

In Diophantine approximation, the situation is better understood. For instance, for $\xi \in \mathbf{R}$, denote by $\lambda(\xi)$ the supremum of all $\lambda$ such that the inequality $|\xi-p / q| \leq$ 
$\max \{|p|,|q|\}^{-\lambda}$ has infinitely many solutions in rational numbers $p / q$ where $p, q \in$ $\mathbf{Z}, q \neq 0$. Then for almost all real numbers $\xi$ we have $\lambda(\xi)=2$, while by Roth's theorem [15], we have also $\lambda(\xi)=2$ for every real, algebraic, irrational number $\xi$.

More generally, the quality of the approximation of a complex number $\xi$ by algebraic numbers of degree at most $n$ can be measured by means of the exponents $w_{n}(\xi)$ and $w_{n}^{*}(\xi)$ introduced by Mahler [14] in 1932 and by Koksma [13] in 1939, respectively, which are defined as follows:

- $w_{n}(\xi)$ denotes the supremum of those real numbers $w$ for which the inequality

$$
0<|P(\xi)| \leq H(P)^{-w}
$$

is satisfied by infinitely many polynomials $P \in \mathbf{Z}[X]$ of degree at most $n$;

- $w_{n}^{*}(\xi)$ denotes the supremum of those real numbers $w^{*}$ for which the inequality

$$
0<|\xi-\alpha| \leq H(\alpha)^{-w^{*}-1}
$$

is satisfied by infinitely many algebraic numbers $\alpha$ of degree at most $n$.

Here, the height $H(P)$ of a polynomial $P \in \mathbf{Z}[X]$ is defined to be the maximum of the absolute values of its coefficients, and the height $H(\alpha)$ of an algebraic number $\alpha$ is defined to be the height of its minimal polynomial (by definition with coprime integer coefficients). The reader is directed to [2] for an overview of the known results on the functions $w_{n}$ and $w_{n}^{*}$.

For every complex number $\xi$ and every integer $n \geq 1$ one has $w_{n}^{*}(\xi) \leq w_{n}(\xi)$, but for every $n \geq 2$, there are complex numbers $\xi$ for which the inequality is strict. Sprindžuk (see his monograph [24]) established in 1965 that for every integer $n \geq$ 1 , we have $w_{n}(\xi)=w_{n}^{*}(\xi)=n$ for almost all real numbers $\xi$ (with respect to the Lebesgue measure on $\mathbf{R}$ ), while $w_{n}(\xi)=w_{n}^{*}(\xi)=\frac{n-1}{2}$ for almost all complex numbers (with respect to the Lebesgue measure on $\mathbf{C}$ ).

Schmidt [20] confirmed that with respect to approximation by algebraic numbers of degree at most $n$, real algebraic numbers of degree larger than $n$ behave like almost all real numbers. Precisely, for every real algebraic number $\xi$ of degree $d$, we have

$$
w_{n}(\xi)=w_{n}^{*}(\xi)=\min \{d-1, n\}
$$

for every integer $n \geq 1$. The $d-1$ in the right-hand side of (1.1) is an immediate consequence of the Liouville inequality. A comparison with Sprindžuk's result gives that if $\xi$ is a real algebraic number of degree $>n$ then $w_{n}(\xi)=w_{n}(\eta)$ for almost all $\eta \in \mathbf{R}$, that is, real algebraic numbers of degree $>n$ are equally well approximable by algebraic numbers of degree at most $n$ as almost all real numbers. 
In this paper we consider the problem to compute $w_{n}(\xi)$ and $w_{n}^{*}(\xi)$ for complex, non-real algebraic numbers $\xi$. It follows again from the Liouville inequality that for complex, non-real algebraic numbers $\xi$ of degree $d \leq n$ one has $w_{n}(\xi)=$ $w_{n}^{*}(\xi)=(d-2) / 2$, but there is no literature about the case where $\xi$ has degree $d>n$. This case is treated in the present paper.

Our results may be summarized as follows. Let $\xi$ be a complex, non-real algebraic number of degree larger than $n$. Then if $n$ is odd, we have $w_{n}(\xi)=$ $w_{n}^{*}(\xi)=\frac{n-1}{2}$, while if $n$ is even we have $w_{n}(\xi)=w_{n}^{*}(\xi) \in\left\{\frac{n-1}{2}, \frac{n}{2}\right\}$. Further, for every even $n$ both cases may occur. In fact, we are able to decide for every positive even integer $n$ and every complex algebraic number $\xi$ whether $w_{n}(\xi)=w_{n}^{*}(\xi)=$ $\frac{n-1}{2}$ or $\frac{n}{2}$, except when $n \geq 6, n+2<\operatorname{deg} \xi \leq 2 n-2,[\mathbf{Q}(\xi): \mathbf{Q}(\xi) \cap \mathbf{R}]=2$, and $1, \xi+\bar{\xi}, \xi \cdot \bar{\xi}$ are linearly independent over $\mathbf{Q}$.

A comparison with Sprindžuk's result for complex numbers mentioned above gives that for every even integer $n \geq 2$ there are complex algebraic numbers $\xi$ of degree $>n$ such that $w_{n}(\xi)>w_{n}(\eta)$ for almost all complex numbers $\eta$. So an important consequence of our results is that in contrast to the real case, for every even integer $n \geq 2$ there are complex algebraic numbers $\xi$ of degree larger than $n$ that are better approximable by algebraic numbers of degree at most $n$ than almost all complex numbers.

We also study how well complex algebraic numbers can be approximated by algebraic integers of bounded degree, and our results support the expectation that complex algebraic numbers which are unusually well approximable by algebraic numbers of degree at most $n$, are unusually badly approximable by algebraic integers of degree at most $n+1$.

We define quantities $\widetilde{w}_{n}(\xi), \widetilde{w}_{n}^{*}(\xi)$ analogously to $w_{n}(\xi), w_{n}^{*}(\xi)$, except that now the approximation is with respect to monic polynomials in $\mathbf{Z}[X]$ of degree at most $n+1$ and complex algebraic integers of degree at most $n+1$, instead of polynomials in $\mathbf{Z}[X]$ of degree at most $n$ and complex algebraic numbers of degree at most $n$. We prove that if $\xi$ is a complex algebraic number of degree larger than $n$, then $\widetilde{w}_{n}(\xi)=\widetilde{w}_{n}^{*}(\xi)=\frac{n-1}{2}$ if $w_{n}(\xi)=\frac{n-1}{2}$, while $\widetilde{w}_{n}(\xi)=\widetilde{w}_{n}^{*}(\xi)=\frac{n-2}{2}$ if $w_{n}(\xi)=\frac{n}{2}$.

Similarly to the case that the number $\xi$ is real algebraic, in our proofs we apply Schmidt's Subspace Theorem and techniques from the geometry of numbers. In this way, we reduce our approximation problem to a purely algebraic problem which does not occur in the real case and which leads to additional difficulties.

Acknowledgements. We are pleased to thank Noriko Hirata-Kohno, Corentin Pontreau and Damien Roy for helpful discussions. The research leading to this paper started with a discussion by both authors at the Erwin Schroedinger Institut in April 2006, in the frame of a research program on Diophantine approximation and heights organized by David Masser, Hans Peter Schlickewei and Wolfgang Schmidt. 


\section{Main results}

The exponents $w_{n}$ and $w_{n}^{*}$ defined in the Introduction measure the quality of algebraic approximation, but do not give any information regarding the number, or the density, of very good approximations. This led the authors of [3] to introduce exponents of uniform Diophantine approximation. For a complex number $\xi$ and an integer $n \geq 1$, we denote by $\hat{w}_{n}(\xi)$ the supremum of those real numbers $w$ for which, for every sufficiently large integer $H$, the inequality

$$
0<|P(\xi)| \leq H^{-w}
$$

is satisfied by an integer polynomial $P$ of degree at most $n$ and height at most $H$.

Khintchine [12] proved that $\hat{w}_{1}(\xi)=1$ for all irrational real numbers $\xi$. Quite unexpectedly, there are real numbers $\xi$ with $\hat{w}_{2}(\xi)>2$. This was established very recently by Roy [16,17] (in fact with $\hat{w}_{2}(\xi)=\frac{3+\sqrt{5}}{2}$ ). However, it is still open whether there exist an integer $n \geq 3$ and a real number $\xi$ such that $\hat{w}_{n}(\xi)>n$.

Our results show that the three functions $w_{n}, w_{n}^{*}$ and $\hat{w}_{n}$ coincide on the set of complex algebraic numbers. Our first result is as follows.

Theorem 2.1. Let $n$ be a positive integer, and $\xi$ a complex, non-real algebraic number of degree $d$. Then

$$
\begin{aligned}
& w_{n}(\xi)=w_{n}^{*}(\xi)=\hat{w}_{n}(\xi)=\frac{d-2}{2} \text { if } d \leq n+1, \\
& w_{n}(\xi)=w_{n}^{*}(\xi)=\hat{w}_{n}(\xi)=\frac{n-1}{2} \text { if } \geq n+2 \text { and } n \text { is odd, } \\
& w_{n}(\xi)=w_{n}^{*}(\xi)=\hat{w}_{n}(\xi) \in\left\{\frac{n-1}{2}, \frac{n}{2}\right\} \text { if } d \geq n+2 \text { and } n \text { is even. }
\end{aligned}
$$

Thus, Theorem 2.1 settles completely the case when $n$ is odd. Henceforth we assume that $n$ is even. In Theorem 2.2 we give some cases where $w_{n}(\xi)=n / 2$ and in Theorem 2.3 some cases where $w_{n}(\xi)=\frac{n-1}{2}$. Unfortunately, we have not been able to compute $w_{n}(\xi)$ in all cases. We denote by $\bar{\alpha}$ the complex conjugate of a complex number $\alpha$.

Theorem 2.2. Let $n$ be an even positive integer and $\xi$ a complex, non-real algebraic number of degree $\geq n+2$. Then $w_{n}(\xi)=w_{n}^{*}(\xi)=\hat{w}_{n}(\xi)=\frac{n}{2}$ in each of the following two cases:

(i) $1, \xi+\bar{\xi}$ and $\xi \cdot \bar{\xi}$ are linearly dependent over $\mathbf{Q}$;

(ii) $\operatorname{deg} \xi=n+2$ and $[\mathbf{Q}(\xi): \mathbf{Q}(\xi) \cap \mathbf{R}]=2$.

One particular special case of (i) is when $\xi=\sqrt{-\alpha}$ for some positive real algebraic number $\alpha$ of degree $\geq \frac{n}{2}+1$. Then $\xi+\bar{\xi}=0$ and so $w_{n}(\xi)=w_{n}^{*}(\xi)=\hat{w}_{n}(\xi)=$ $n / 2$.

We do not know whether Theorem 2.2 covers all cases where $w_{n}(\xi)=\frac{n}{2}$. We now give some cases where $w_{n}(\xi)=\frac{n-1}{2}$. 
Theorem 2.3. Let again $n$ be an even positive integer and $\xi$ a complex, non-real algebraic number of degree $\geq n+2$. Then $w_{n}(\xi)=w_{n}^{*}(\xi)=\hat{w}_{n}(\xi)=\frac{n-1}{2}$ in each of the following two cases:

(i) $[\mathbf{Q}(\xi): \mathbf{Q}(\xi) \cap \mathbf{R}] \geq 3$;

(ii) $\operatorname{deg} \xi>2 n-2$ and $1, \xi+\bar{\xi}, \xi \cdot \bar{\xi}$ are linearly independent over $\mathbf{Q}$.

For $n=2,4$ we have $2 n-2 \leq n+2$, so in that case Theorems 2.2 and 2.3 cover all complex algebraic numbers $\xi$. Further, for $n=2$, case (ii) of Theorem 2.2 is implied by case (i). This leads to the following corollary.

Corollary 2.4. Let $\xi$ be a complex, non-real algebraic number.

(i) If $\xi$ has degree $>2$, then

$$
\begin{aligned}
& w_{2}(\xi)=w_{2}^{*}(\xi)=\hat{w}_{2}(\xi)=1 \text { if } 1, \xi+\bar{\xi}, \xi \cdot \bar{\xi} \text { are linearly dependent over } \mathbf{Q} \\
& w_{2}(\xi)=w_{2}^{*}(\xi)=\hat{w}_{2}(\xi)=\frac{1}{2} \text { otherwise. }
\end{aligned}
$$

(ii) If $\xi$ has degree $>4$, then

$$
\begin{aligned}
& w_{4}(\xi)=w_{4}^{*}(\xi)=\hat{w}_{4}(\xi)=2 \text { if } 1, \xi+\bar{\xi}, \xi \cdot \bar{\xi} \text { are linearly dependent over } \mathbf{Q} \\
& \quad \text { or if } \operatorname{deg} \xi=6 \text { and }[\mathbf{Q}(\xi): \mathbf{Q}(\xi) \cap \mathbf{R}]=2, \\
& w_{4}(\xi)=w_{4}^{*}(\xi)=\hat{w}_{4}(\xi)=\frac{3}{2} \text { otherwise. }
\end{aligned}
$$

Theorems 2.1, 2.2, 2.3 and Corollary 2.4 allow us to determine $w_{n}(\xi), w_{n}^{*}(\xi), \hat{w}_{n}(\xi)$ for every positive integer $n$ and every complex, non-real algebraic number $\xi$, with the exception of the following case:

$n$ is an even integer with $n \geq 6, \xi$ is a complex algebraic number such that $n+2<$ $\operatorname{deg} \xi \leq 2 n-2,[\mathbf{Q}(\xi): \mathbf{Q}(\xi) \cap \mathbf{R}]=2$ and $1, \xi+\bar{\xi}, \xi \cdot \bar{\xi}$ are linearly independent over $\mathbf{Q}$.

We deduce Theorems 2.1, 2.2, 2.3 from Theorem 2.5 below. To state the latter, we have to introduce some notation. For $n \in \mathbf{Z}_{>0}, \xi \in \mathbf{C}^{*}, \mu \in \mathbf{C}^{*}$, define the $\mathbf{Q}$-vector space

$$
V_{n}(\mu, \xi):=\{f \in \mathbf{Q}[X]: \operatorname{deg} f \leq n, \mu f(\xi) \in \mathbf{R}\},
$$

and for $n \in \mathbf{Z}_{>0}, \xi \in \mathbf{C}^{*}$ denote by $t_{n}(\xi)$ the maximum over $\mu$ of the dimensions of these spaces, i.e.,

$$
t_{n}(\xi):=\max \left\{\operatorname{dim}_{\mathbf{Q}} V_{n}(\mu, \xi): \mu \in \mathbf{C}^{*}\right\} .
$$

It is clear that $t_{n}(\xi) \leq n+1$ and $t_{n}(\xi)=n+1$ if and only if $\xi \in \mathbf{R}$. 
Theorem 2.5. Let $n$ be a positive integer and $\xi$ a complex, non-real algebraic number of degree $>n$. Then

$$
w_{n}(\xi)=w_{n}^{*}(\xi)=\hat{w}_{n}(\xi)=\max \left\{\frac{n-1}{2}, t_{n}(\xi)-1\right\}
$$

The proof of Theorem 2.5 is based on Schmidt's Subspace Theorem and geometry of numbers. It should be noted that Theorem 2.5 reduces the problem to determine how well $\xi$ can be approximated by algebraic numbers of degree at most $n$ to the algebraic problem to compute $t_{n}(\xi)$. We deduce Theorems $2.1,2.2$ and 2.3 by combining Theorem 2.5 with some properties of the quantity $t_{n}(\xi)$ proved below.

\section{Approximation by algebraic integers}

In view of a transference lemma relating uniform homogeneous approximation to inhomogeneous approximation (see [4]), for any integer $n \geq 2$, the real numbers $\xi$ with $\hat{w}_{n}(\xi)>n$ are good candidates for being unexpectedly badly approximable by algebraic integers of degree less than or equal to $n+1$. This has been confirmed by Roy [18] for the case $n=2$. Namely, in [17] he proved that there exist real numbers $\xi$ with $\hat{w}_{2}(\xi)=\frac{3+\sqrt{5}}{2}>2$, and in [18] he used this to prove that there exist real numbers $\xi$ with the property that $|\xi-\alpha| \gg H(\alpha)^{-(3+\sqrt{5}) / 2}$ for every algebraic integer $\alpha$ of degree at most 3. By a result of Davenport and Schmidt [9], the exponent $\frac{3+\sqrt{5}}{2}$ is optimal. On the other hand Bugeaud and Teulie [5] proved that for every $\kappa<3$ and almost all $\xi \in \mathbf{R}$, the inequality $|\xi-\alpha|<H(\alpha)^{-\kappa}$ has infinitely many solutions in algebraic integers of degree 3 .

Analogously to the real case one should expect that complex numbers $\xi$ with $\hat{w}_{n}(\xi)>\frac{n-1}{2}$ are unusually badly approximable by algebraic integers of degree at most $n+1$. In Theorem 3.1 below we confirm this for complex algebraic numbers.

We introduce the following quantities for complex numbers $\xi$ and integers $n \geq 1$ :

- $\widetilde{w}_{n}(\xi)$ denotes the supremum of those real numbers $\widetilde{w}$ such that

$$
0<|P(\xi)| \leq H(P)^{-\widetilde{w}}
$$

is satisfied by infinitely many monic polynomials $P \in \mathbf{Z}[X]$ of degree at most $n+1$;

- $\widetilde{w}_{n}^{*}(\xi)$ denotes the supremum of those real numbers $\widetilde{w}^{*}$ for which

$$
0<|\xi-\alpha| \leq H(\alpha)^{-\widetilde{w}^{*}-1}
$$

holds for infinitely many algebraic integers of degree at most $n+1$; 
- $\hat{\widetilde{w}}_{n}(\xi)$ denotes the supremum of those real numbers $\widetilde{w}$ with the property that for every sufficiently large real $H$, there exists a monic integer polynomial $P$ of degree at most $n+1$ and height at most $H$ such that

$$
0<|P(\xi)| \leq H^{-\widetilde{w}}
$$

It is known that every real algebraic number $\xi$ of degree $d$ satisfies

$$
\widetilde{w}_{n}(\xi)=\widetilde{w}_{n}^{*}(\xi)=\hat{\widetilde{w}}_{n}(\xi)=\min \{d-1, n\}
$$

for every integer $n$ (see [2, 22]). Furthermore, methods developed by Bugeaud and Teulié [5] and Roy and Waldschmidt [19] allow one to show that for every positive integer $n$ we have

$$
\begin{aligned}
& \widetilde{w}_{n}(\xi)=\widetilde{w}_{n}^{*}(\xi)=\hat{\widetilde{w}}_{n}(\xi)=n \text { for almost all } \xi \in \mathbf{R}, \\
& \widetilde{w}_{n}(\xi)=\widetilde{w}_{n}^{*}(\xi)=\hat{\widetilde{w}}_{n}(\xi)=\frac{n-1}{2} \text { for almost all } \xi \in \mathbf{C} .
\end{aligned}
$$

We show that for every positive integer $n$ the functions $\widetilde{w}_{n}, \widetilde{w}_{n}^{*}, \hat{\widetilde{w}}_{n}$ coincide on the complex algebraic numbers and, moreover, that a complex algebraic number $\xi$ is unusually badly approximable by algebraic integers of degree at most $n+1$ (i.e., has $\left.\widetilde{w}_{n}(\xi)=\widetilde{w}_{n}^{*}(\xi)=\hat{\widetilde{w}}_{n}(\xi)<\frac{n-1}{2}\right)$ if and only if it is unusually well approximable by algebraic numbers of degree at most $n$ (i.e., has $w_{n}(\xi)=w_{n}^{*}(\xi)=\hat{w}_{n}(\xi)>\frac{n-1}{2}$ ). More precisely, we prove the following.

Theorem 3.1. Let $n$ be a positive integer and $\xi$ a complex, non-real algebraic number of degree $d$. Then

$$
\begin{aligned}
& \widetilde{w}_{n}(\xi)=\widetilde{w}_{n}^{*}(\xi)=\hat{\widetilde{w}}_{n}(\xi)=\frac{d-2}{2} \text { if } d \leq n+1 \\
& \widetilde{w}_{n}(\xi)=\widetilde{w}_{n}^{*}(\xi)=\hat{\widetilde{w}}_{n}(\xi)=\frac{n-1}{2} \text { if } d \geq n+2 \text { and } n \text { is odd }, \\
& \widetilde{w}_{n}(\xi)=\widetilde{w}_{n}^{*}(\xi)=\hat{\widetilde{w}}_{n}(\xi) \in\left\{\frac{n-2}{2}, \frac{n-1}{2}\right\} \text { if } d \geq n+2 \text { and } n \text { is even. }
\end{aligned}
$$

Moreover, if $d \geq n+2$ and $n$ is even then

$$
\widetilde{w}_{n}(\xi)=\widetilde{w}_{n}^{*}(\xi)=\hat{\widetilde{w}}_{n}(\xi)=\frac{n-2}{2} \Longleftrightarrow w_{n}(\xi)=w_{n}^{*}(\xi)=\hat{w}_{n}(\xi)=\frac{n}{2}
$$

Combining Theorem 3.1 with Corollary 2.4, we get at once the following statement. 
Corollary 3.2. Let $\xi$ be a complex, non-real algebraic number.

(i) If $\xi$ has degree $>2$, then

$$
\begin{aligned}
& \widetilde{w}_{2}(\xi)=\widetilde{w}_{2}^{*}(\xi)=\hat{\widetilde{w}}_{2}(\xi)=0 \text { if } 1, \xi+\bar{\xi}, \xi \cdot \bar{\xi} \text { are linearly dependent over } \mathbf{Q} \\
& \widetilde{w}_{2}(\xi)=\widetilde{w}_{2}^{*}(\xi)=\hat{\widetilde{w}}_{2}(\xi)=\frac{1}{2} \text { otherwise. }
\end{aligned}
$$

(ii) If $\xi$ has degree $>4$, then

$$
\begin{array}{ll}
\widetilde{w}_{4}(\xi)=\widetilde{w}_{4}^{*}(\xi)=\hat{\widetilde{w}}_{4}(\xi)=1 \quad \begin{array}{l}
\text { if } 1, \xi+\bar{\xi}, \xi \cdot \bar{\xi} \text { are linearly dependent over } \mathbf{Q} \\
\text { or if } \operatorname{deg} \xi=6 \text { and }[\mathbf{Q}(\xi): \mathbf{Q}(\xi) \cap \mathbf{R}]=2,
\end{array} \\
\widetilde{w}_{4}(\xi)=\widetilde{w}_{4}^{*}(\xi)=\hat{\widetilde{w}}_{4}(\xi)=\frac{3}{2} \quad \begin{array}{l}
\text { otherwise. }
\end{array}
\end{array}
$$

\section{Deduction of Theorem 2.1 from Theorem 2.5}

For every positive integer $m$ we define the $\mathbf{Q}$-vector space

$$
W_{m}:=\{f \in \mathbf{Q}[X]: \operatorname{deg} f \leq m\}
$$

and for any subset $S$ of the polynomial ring $\mathbf{Q}[X]$ and any polynomial $g \in \mathbf{Q}[X]$, we define the set $g \cdot S:=\{g f: f \in S\}$.

In this section, $n$ is a positive integer, and $\xi$ a complex, non-real algebraic number of degree $d>n$. We prove some lemmata about the quantity $t_{n}(\xi)$ which in combination with Theorem 2.5 will imply Theorem 2.1. Choose $\mu_{0} \in \mathbf{C}^{*}$ such that $\operatorname{dim} V_{n}\left(\mu_{0}, \xi\right)=t_{n}(\xi)$.

Lemma 4.1. Let $\mu \in \mathbf{C}^{*}$ be such that $\operatorname{dim} V_{n}(\mu, \xi)>\frac{n+1}{2}$. Then $V_{n}(\mu, \xi)=$ $V_{n}\left(\mu_{0}, \xi\right)$.

Proof. Our assumption on $\mu$ clearly implies that $t_{n}(\xi)>\frac{n+1}{2}$. Both vector spaces $V_{n}(\mu, \xi), V_{n}\left(\mu_{0}, \xi\right)$ are contained in the same $n+1$-dimensional vector space, hence they have non-zero intersection. Let $f_{1} \in \mathbf{Q}[X]$ be a non-zero polynomial lying in both spaces and put $\mu_{1}:=f_{1}(\xi)^{-1}$. Then $\mu_{1} / \mu \in \mathbf{R}, \mu_{1} / \mu_{0} \in \mathbf{R}$, hence $V_{n}(\mu, \xi)=V_{n}\left(\mu_{1}, \xi\right)=V_{n}\left(\mu_{0}, \xi\right)$.

Lemma 4.2. Suppose that $t_{n}(\xi)>\frac{n+1}{2}$. Then

(i) $W_{n+1}$ is the direct sum of the $\mathbf{Q}$-vector spaces $V_{n}\left(\mu_{0}, \xi\right)$ and $X \cdot V_{n}\left(\mu_{0}, \xi\right)$.

(ii) $n$ is even, $t_{n}(\xi)=\frac{n+2}{2}$. 
Proof. Suppose that $V_{n}\left(\mu_{0}, \xi\right) \cap X \cdot V_{n}\left(\mu_{0}, \xi\right) \neq\{0\}$. Choose a non-zero polynomial $f$ in the intersection of both spaces. Then $f=X g$ where $g \in V_{n}\left(\mu_{0}, \xi\right)$. Hence

$$
\xi=\frac{f(\xi)}{g(\xi)}=\frac{\mu_{0} f(\xi)}{\mu_{0} g(\xi)} \in \mathbf{R},
$$

which is against our assumption. Therefore, $V_{n}\left(\mu_{0}, \xi\right) \cap X \cdot V_{n}\left(\mu_{0}, \xi\right)=\{0\}$. From our assumption on $\xi$ it follows that $t_{n}(\xi) \geq \frac{n+2}{2}$. Further, both $V_{n}\left(\mu_{0}, \xi\right)$ and $X \cdot V_{n}\left(\mu_{0}, \xi\right)$ are linear subspaces of $W_{n+1}$. Hence by comparing dimensions,

$$
2 \cdot \frac{n+2}{2} \leq 2 t_{n}(\xi)=\operatorname{dim}\left(V_{n}\left(\mu_{0}, \xi\right)+X \cdot V_{n}\left(\mu_{0}, \xi\right)\right) \leq \operatorname{dim} W_{n+1}=n+2 .
$$

This implies (i) and (ii).

Lemma 4.3. Let $\xi$ be a complex, non-real algebraic number of degree $d>1$. Then $t_{d-1}(\xi) \leq \frac{d}{2}$.

Proof. Choose $\mu_{0} \in \mathbf{C}^{*}$ such that $\operatorname{dim} V_{d-1}\left(\mu_{0}, \xi\right)=t_{d-1}(\xi)$. Pick a non-zero polynomial $f_{0} \in V_{d-1}\left(\mu_{0}, \xi\right)$. Then for every $f \in V_{d-1}\left(\mu_{0}, \xi\right)$ we have $\frac{f(\xi)}{f_{0}(\xi)}=$ $\frac{\mu_{0} f(\xi)}{\mu_{0} f_{0}(\xi)} \in \mathbf{Q}(\xi) \cap \mathbf{R}$. For linearly independent polynomials $f \in \mathbf{Q}[X]$ of degree at most $d-1=\operatorname{deg} \xi-1$, the corresponding quantities $f(\xi) / f_{0}(\xi)$ are linearly independent over $\mathbf{Q}$. Hence $t_{d-1}(\xi) \leq[\mathbf{Q}(\xi) \cap \mathbf{R}: \mathbf{Q}] \leq \frac{d}{2}$.

In the proof of Theorem 2.1 we use the following observations.

Lemma 4.4. Let $\xi$ be a complex number and $n$ a positive integer. Then

(i) $w_{n}^{*}(\xi) \leq w_{n}(\xi)$,

(ii) $\hat{w}_{n}(\xi) \leq w_{n}(\xi)$.

Proof. If $\alpha$ is an algebraic number of degree $n$ with minimal polynomial $P \in \mathbf{Z}[X]$, we have $|P(\xi)| \ll H(P) \cdot \min \{1,|\alpha-\xi|\}$, where the implied constant depends only on $\xi$ and on $n$. This implies (i). If for some $w \in \mathbf{R}$ there exists $H_{0}$ such that for every $H \geq H_{0}$ there exists an integer polynomial $P$ of degree at most $n$ with $0<|P(\xi)| \leq H^{-w}, H(P) \leq H$, then clearly, there are infinitely many integer polynomials $P$ of degree at most $n$ such that $0<|P(\xi)| \leq H(P)^{-w}$. This implies (ii).

Proof of Theorem 2.1. Constants implied by $\ll$ and $\gg$ depend only on $n, \xi$. We first prove (2.1). Assume that $d \leq n+1$. In view of Lemma 4.4, it suffices to prove that

$$
w_{n}(\xi) \leq \frac{d-2}{2}, w_{n}^{*}(\xi) \geq \frac{d-2}{2}, \quad \hat{w}_{n}(\xi) \geq \frac{d-2}{2} .
$$

To prove the former, denote by $\xi^{(1)}, \ldots, \xi^{(d)}$ the conjugates of $\xi$, where $\xi^{(1)}=\xi$, $\xi^{(2)}=\bar{\xi}$. For some $a \in \mathbf{Z}_{>0}$, the polynomial $Q:=a \prod_{i=1}^{d}\left(X-\xi^{(i)}\right)$ has integer 
coefficients, and for any polynomial $P \in \mathbf{Z}[X]$ of degree at most $n$ with $P(\xi) \neq 0$, the resultant $R(P, Q)=a^{n} \prod_{i=1}^{d} P\left(\xi^{(i)}\right)$ is a non-zero rational integer. This gives the Liouville inequality

$$
|P(\xi)|^{2}=|P(\xi) P(\bar{\xi})| \gg \frac{|R(P, Q)|}{\left|P\left(\xi^{(3)}\right) \cdots P\left(\xi^{(d)}\right)\right|} \gg H(P)^{2-d} .
$$

Consequently, $w_{n}(\xi) \leq \frac{d-2}{2}$.

By Theorem 2.5 with $n=d-1$ and by Lemma 4.3 we have $w_{d-1}^{*}(\xi)=$ $\hat{w}_{d-1}(\xi)=\frac{d-2}{2}$. Using that $w_{n}^{*}(\xi), \hat{w}_{n}(\xi)$ are non-decreasing in $n$, we obtain that for $n \geq d-1$,

$$
w_{n}^{*}(\xi) \geq w_{d-1}^{*}(\xi)=\frac{d-2}{2}, \quad \hat{w}_{n}(\xi) \geq \hat{w}_{d-1}(\xi)=\frac{d-2}{2} .
$$

This completes the proof of (2.1).

Statements (2.2), (2.3) follow immediately by combining Theorem 2.5 with part (ii) of Lemma 4.2. This completes the proof of Theorem 2.1.

\section{Deduction of Theorem 2.2 from Theorem 2.5}

To deduce Theorem 2.2 from Theorem 2.5, we prove again the necessary properties for the quantity $t_{n}(\xi)$ defined by (2.5).

Lemma 5.1. Assume that $n$ is even, and that $\xi$ is a complex, non-real algebraic number of degree $>n$ such that $1, \xi+\bar{\xi}$ and $\xi \cdot \bar{\xi}$ are linearly dependent over $\mathbf{Q}$. Then

$$
t_{n}(\xi)=\frac{n+2}{2} .
$$

Proof. We use the easy observation that $t_{n}(\xi+c)=t_{n}(\xi)$ for any $c \in \mathbf{Q}$.

Put $\beta:=\xi+\bar{\xi}, \gamma:=\xi \cdot \bar{\xi}$. Our assumption on $\xi$ implies that either $\beta \in \mathbf{Q}$, or $\gamma=a+b \beta$ for some $a, b \in \mathbf{Q}$. By our observation, the first case can be reduced to $\beta=0$ by replacing $\xi$ by $\xi-\frac{1}{2} \beta$. Then $\xi=\sqrt{-\gamma}$ with $\gamma>0$. Likewise, the second case can be reduced to $\gamma=a \in \mathbf{Q}$ by replacing $\xi$ by $\xi-b$. Then $\xi=\frac{1}{2}\left(\beta \pm \sqrt{\beta^{2}-4 a}\right)$ with $a \in \mathbf{Q}$ and $a>\beta^{2} / 4$.

Case I. $\xi=\sqrt{-\gamma}$ with $\gamma>0$.

In this case,

$V_{n}(1, \xi)=\{f \in \mathbf{Q}[X]: \operatorname{deg} f \leq n, f(\xi) \in \mathbf{R}\}=\left\{\sum_{i=0}^{n / 2} c_{i} X^{2 i}: c_{0}, \ldots, c_{n / 2} \in \mathbf{Q}\right\}$.

So $t_{n}(\xi) \geq \operatorname{dim} V_{n}(1, \xi)=\frac{n+2}{2}$. Hence by Lemma 4.2 we have $t_{n}(\xi)=\frac{n+2}{2}$. 
Case II. $\gamma=\xi \cdot \bar{\xi}=a \in \mathbf{Q}^{*}$.

Put $\mu:=\xi^{-n / 2}$. Then for a polynomial $f=\sum_{i=0}^{n} c_{i} X^{i} \in \mathbf{Q}[X]$ we have, recalling our assumption that $\xi$ has degree larger than $n$,

$$
\begin{aligned}
\mu f(\xi) \in \mathbf{R} & \Longleftrightarrow \xi^{-n / 2} f(\xi)=\bar{\xi}^{(-n / 2)} f(\bar{\xi}) \Longleftrightarrow \xi^{-n / 2} f(\xi)=(a / \xi)^{-n / 2} f(a / \xi) \\
& \Longleftrightarrow a^{n / 2} f(\xi)=\xi^{n} f(a / \xi) \Longleftrightarrow a^{n / 2} f(X)=X^{n} f(a / X) \\
& \Longleftrightarrow a^{n / 2} c_{i}=a^{n-i} c_{n-i} \text { for } i=0, \ldots, n .
\end{aligned}
$$

This implies $t_{n}(\xi) \geq \operatorname{dim} V_{n}(\mu, \xi)=\frac{n+2}{2}$. Hence $t_{n}(\xi)=\frac{n+2}{2}$ in view of Lemma 4.2.

Lemma 5.2. Let $n$ be an even positive integer, and $\xi$ a complex algebraic number of degree $n+2$. Suppose that $[\mathbf{Q}(\xi): \mathbf{Q}(\xi) \cap \mathbf{R}]=2$. Then

$$
t_{n}(\xi)=\frac{n+2}{2} .
$$

Proof. Write $k:=n / 2$. Then $\mathbf{Q}(\xi) \cap \mathbf{R}$ has degree $k+1$. We prove that there exists $\mu \in \mathbf{Q}(\xi)^{*}$ such that $\operatorname{dim} V_{n}(\mu, \xi) \geq k+1=\frac{n+2}{2}$. Then from Lemma 4.2 it follows that $t_{n}(\xi)=\frac{n+2}{2}$.

Let $\left\{\omega_{1}, \ldots, \omega_{k+1}\right\}$ be a $\mathbf{Q}$-basis of $\mathbf{Q}(\xi) \cap \mathbf{R}$.Then $\omega_{1}, \ldots, \omega_{k+1}, \xi \omega_{1}, \ldots, \xi \omega_{k+1}$ form a $\mathbf{Q}$-basis of $\mathbf{Q}(\xi)$, every element of $\mathbf{Q}(\xi)$ can be expressed uniquely as a $\mathbf{Q}$ linear combination of these numbers, and a number in $\mathbf{Q}(\xi)$ thus expressed belongs to $\mathbf{Q}(\xi) \cap \mathbf{R}$ if and only if its coefficients with respect to $\xi \omega_{1}, \ldots, \xi \omega_{k+1}$ are 0 .

For $i, j=0, \ldots, 2 k+1$ we have

$$
\xi^{i+j}=\sum_{l=1}^{k+1} a_{i j}^{(l)} \omega_{l}+\sum_{l=1}^{k+1} b_{i j}^{(l)} \xi \omega_{l} \quad \text { with } a_{i j}^{(l)}, b_{i j}^{(l)} \in \mathbf{Q} .
$$

Write $\mu \in \mathbf{Q}(\xi)$ as $\mu=\sum_{i=0}^{2 k+1} u_{i} \xi^{i}$ with $u_{0}, \ldots, u_{2 k+1} \in \mathbf{Q}$ and write $f \in$ $V_{n}(\mu, \xi)$ as $f=\sum_{j=0}^{2 k} x_{j} X^{j}$ with $x_{0}, \ldots, x_{2 k} \in \mathbf{Q}$. Then

$$
\mu f(\xi)=\sum_{l=1}^{k+1} \omega_{l}\left\{\sum_{j=0}^{2 k}\left(\sum_{i=0}^{2 k+1} a_{i j}^{(l)} u_{i}\right) x_{j}\right\}+\sum_{l=1}^{k+1} \xi \omega_{l}\left\{\sum_{j=0}^{2 k}\left(\sum_{i=0}^{2 k+1} b_{i j}^{(l)} u_{i}\right) x_{j}\right\} .
$$

So $f=\sum_{j=0}^{2 k} x_{j} X^{j} \in V_{n}(\mu, \xi)$, i.e., $\mu f(\xi) \in \mathbf{Q}(\xi) \cap \mathbf{R}$, if and only if

$$
L_{\mu}^{(l)}(\mathbf{x}):=\sum_{j=0}^{2 k}\left(\sum_{i=0}^{2 k+1} b_{i j}^{(l)} u_{i}\right) x_{j}=0 \quad \text { for } l=1, \ldots, k+1,
$$

where $\mathbf{x}=\left(x_{0}, \ldots, x_{2 k}\right)$. 
We choose $\mu \in \mathbf{Q}(\xi)^{*}$ to make one of the linear forms in (5.1), for instance $L_{\mu}^{(k+1)}$, vanish identically. This amounts to choosing a non-zero vector $\mathbf{u}=\left(u_{0}, \ldots, u_{2 k+1}\right) \in \mathbf{Q}^{2 k+2}$ such that

$$
\sum_{i=0}^{2 k+1} b_{i j}^{(k+1)} u_{i}=0 \text { for } j=0, \ldots, 2 k .
$$

This is possible since a system of $2 k+1$ linear equations in $2 k+2$ unknowns has a non-trivial solution. Thus, (5.1) becomes a system of $k$ equations in $2 k+1$ unknowns over $\mathbf{Q}$, and the solution space of this system has dimension at least $k+1$. Consequently, $V_{n}(\mu, \xi)$ has dimension at least $k+1=\frac{n+2}{2}$. This proves Lemma 5.2.

Now Theorem 2.2 follows at once by combining Theorem 2.5 with Lemmata 5.1 and 5.2.

\section{Deduction of Theorem 2.3 from Theorem 2.5}

We prove some results about the quantity $t_{n}(\xi)$ which, in combination with Theorem 2.5, will yield Theorem 2.3.

Lemma 6.1. Let $n$ be an even positive integer and $\xi$ a complex, non-real algebraic number of degree $>n$. Assume that $t_{n}(\xi)>\frac{n+1}{2}$.

(i) $[\mathbf{Q}(\xi): \mathbf{Q}(\xi) \cap \mathbf{R}]=2$.

(ii) If moreover $\operatorname{deg} \xi>2 n-2$, then $1, \xi+\bar{\xi}, \xi \cdot \bar{\xi}$ are linearly dependent over $\mathbf{Q}$.

Proof. Put $\beta:=\xi+\bar{\xi}, \gamma:=\xi \cdot \bar{\xi}$. Choose $\mu_{0}$ such that $\operatorname{dim} V_{n}\left(\mu_{0}, \xi\right)=t_{n}(\xi)$. By part (i) of Lemma 4.2, every polynomial in $\mathbf{Q}[X]$ of degree at most $n+1$ can be expressed uniquely as a sum of a polynomial in $V_{n}\left(\mu_{0}, \xi\right)$ and a polynomial in $X \cdot V_{n}\left(\mu_{0}, \xi\right)$. In particular, for every non-zero polynomial $f \in V_{n}\left(\mu_{0}, \xi\right)$ of degree $\leq n-1$, there are polynomials $g, h \in V_{n}\left(\mu_{0}, \xi\right)$, uniquely determined by $f$, such that

$$
X^{2} f=X g+h .
$$

This implies that $\xi$ is a zero of the polynomial $X^{2}-(g(\xi) / f(\xi)) X-(h(\xi) / f(\xi))$. On the other hand, there is a unique monic quadratic polynomial with real coefficients having $\xi$ as a zero, namely $X^{2}-\beta X+\gamma$, and

$$
\frac{g(\xi)}{f(\xi)}=\frac{\mu_{0} g(\xi)}{\mu_{0} f(\xi)} \in \mathbf{R}, \quad \frac{h(\xi)}{f(\xi)}=\frac{\mu_{0} h(\xi)}{\mu_{0} f(\xi)} \in \mathbf{R} .
$$

Therefore,

$$
\frac{g(\xi)}{f(\xi)}=\beta, \quad \frac{h(\xi)}{f(\xi)}=-\gamma
$$

So $\beta, \gamma \in \mathbf{Q}(\xi) \cap \mathbf{R}$. This implies (i). 
To prove (ii), we proceed by induction on $n$. First let $n=2$. By assumption, there is $\mu_{0} \in \mathbf{C}^{*}$ such that $V_{2}\left(\mu_{0}, \xi\right)$ has dimension larger than 1 . This means that there are non-zero polynomials $f_{1}, f_{2} \in V_{2}\left(\mu_{0}, \xi\right)$ with $\operatorname{deg} f_{1}<\operatorname{deg} f_{2} \leq 2$. We have $f_{1}(\xi) f_{2}(\bar{\xi}) \in\left(\mu_{0} \overline{\mu_{0}}\right)^{-1} \mathbf{R}=\mathbf{R}$, hence

$$
f_{1}(\xi) f_{2}(\bar{\xi})-f_{1}(\bar{\xi}) f_{2}(\xi)=0 .
$$

First suppose that $f_{2}$ has degree 1 . Then $f_{1}$ has degree 0 , therefore, $f_{1}=c_{1}$, $f_{2}=c_{2}+c_{3} X$ with $c_{1} c_{3} \neq 0$. Hence

$$
0=f_{1}(\xi) f_{2}(\bar{\xi})-f_{1}(\bar{\xi}) f_{2}(\xi)=c_{1} c_{3}(\bar{\xi}-\xi),
$$

which is impossible since $\xi \notin \mathbf{R}$. Now suppose that $f_{2}$ has degree 2 . Then $f_{1}=$ $c_{1}+c_{2} X, f_{2}=c_{3}+c_{4} X+c_{5} X^{2}$ with $c_{1}, \ldots, c_{5} \in \mathbf{Q}$, hence

$$
0=f_{1}(\xi) f_{2}(\bar{\xi})-f_{1}(\bar{\xi}) f_{2}(\xi)=(\bar{\xi}-\xi)\left(c_{1} c_{4}-c_{2} c_{3}+c_{1} c_{5} \beta+c_{2} c_{5} \gamma\right) .
$$

We have $\left(c_{1}, c_{2}\right) \neq(0,0)$ since $f_{1} \neq 0$, while $c_{5} \neq 0$ since $f_{2}$ has degree 2 , and further $\xi \notin \mathbf{R}$. Hence $1, \beta, \gamma$ are $\mathbf{Q}$-linearly dependent.

Now let $n$ be an even integer with $n \geq 4$. Assume part (ii) of Lemma 6.1 is true if $n$ is replaced by any positive even integer smaller than $n$. There is $\mu_{0} \in \mathbf{C}^{*}$ such that $\operatorname{dim} V_{n}\left(\mu_{0}, \xi\right)=: t>\frac{n+1}{2}$. Let $f_{1}, \ldots, f_{t}$ be a basis of $V_{n}\left(\mu_{0}, \xi\right)$ with $\operatorname{deg} f_{1}<\operatorname{deg} f_{2}<\cdots<\operatorname{deg} f_{t} \leq n$. So in particular, deg $f_{t-1} \leq n-1$.

First assume that $a:=\operatorname{gcd}\left(f_{1}, \ldots, f_{t-1}\right)$ is a polynomial of degree at least 1. Let $\tilde{f}_{i}:=f_{i} / a$ for $i=1, \ldots, t-1$. Put $\tilde{\mu}_{0}:=\mu_{0} a(\xi)$. Then $\tilde{f}_{1}, \ldots, \tilde{f}_{t-1}$ are linearly independent polynomials of degree at most $n-2$ with $\tilde{\mu_{0}} \tilde{f}_{i}(\xi) \in \mathbf{R}$ for $i=1, \ldots, t-1$. Hence

$$
t_{n-2}(\xi) \geq \operatorname{dim} V_{n-2}\left(\tilde{\mu_{0}}, \xi\right) \geq t-1>\frac{(n-2)+1}{2} .
$$

So by the induction hypothesis, $1, \beta, \gamma$ are linearly dependent over $\mathbf{Q}$.

Now assume that $\operatorname{gcd}\left(f_{1}, \ldots, f_{t-1}\right)=1$. By $(6.1)$, for $i=1, \ldots, t-1$ there are polynomials $g_{i}, h_{i} \in V_{n}\left(\mu_{0}, \xi\right)$ such that $X^{2} f_{i}=X g_{i}+h_{i}$ for $i=1, \ldots, t-1$ and by (6.2) we have

$$
\frac{g_{i}(\xi)}{f_{i}(\xi)}=\beta, \quad \frac{h_{i}(\xi)}{f_{i}(\xi)}=-\gamma \quad \text { for } i=1, \ldots, t-1 .
$$

The polynomials $h_{i}$ are all divisible by $X$. Therefore, $\xi$ is a common zero of the polynomials

$$
f_{i} \cdot \frac{h_{j}}{X}-f_{j} \cdot \frac{h_{i}}{X} \quad(1 \leq i, j \leq t-1) .
$$

Each of these polynomials has degree at most $2 n-2$ and, by assumption, $\xi$ has degree $>2 n-2$. Therefore, these polynomials are all identically 0 . Since by 
assumption $\operatorname{gcd}\left(f_{1}, \ldots, f_{t-1}\right)=1$, this implies that there is a polynomial $a \in$ $\mathbf{Q}[X]$ with $h_{i} / X=a f_{i}$ for $i=1, \ldots, t-1$.

Now $a$ cannot be equal to 0 since otherwise $\gamma=\xi \cdot \bar{\xi}$ would be 0 which is impossible. Further, $a$ cannot be a constant $c \in \mathbf{Q}^{*}$ since otherwise, we would have $\xi=h_{i}(\xi) / c f_{i}(\xi)=-\gamma / c \in \mathbf{R}$ which is impossible. Hence $a$ has degree at least 1 . But then $\operatorname{deg} f_{i} \leq \operatorname{deg} h_{i}-2 \leq n-2$ for $i=1, \ldots, t-1$. This implies

$$
t_{n-2}(\xi) \geq \operatorname{dim} V_{n-2}(\mu, \xi) \geq t-1>\frac{n-2+1}{2} .
$$

Now again the induction hypothesis can be applied, and we infer that $1, \beta, \gamma$ are linearly dependent over $\mathbf{Q}$. This completes our proof.

Theorem 2.3 follows at once by combining Theorem 2.5 with Lemma 6.1 .

\section{Consequences of the parametric subspace theorem}

In this section we have collected some applications of the Parametric Subspace Theorem which are needed in both the proofs of Theorem 2.5 and Theorem 3.1. Our arguments are a routine extension of [23, Chapter VI, Sections 1, 2, Schmidt's Lecture Notes], but for lack of a convenient reference we have included the proofs.

We start with some notation. For a linear form $L=\sum_{i=1}^{n} \alpha_{i} X_{i}$ with complex coefficients, we write $\operatorname{Re}(L):=\sum_{i=1}^{n}\left(\operatorname{Re} \alpha_{i}\right) X_{i}$ and $\operatorname{Im}(L):=\sum_{i=1}^{n}\left(\operatorname{Im} \alpha_{i}\right) X_{i}$. For a linear subspace $U$ of $\mathbf{Q}^{n}$, we denote by $\mathbf{R} U$ the $\mathbf{R}$-linear subspace of $\mathbf{R}^{n}$ generated by $U$. We say that linear forms $L_{1}, \ldots, L_{s}$ in $X_{1}, \ldots, X_{n}$ with complex coefficients are linearly dependent on a linear subspace $U$ of $\mathbf{Q}^{n}$ if there are complex numbers $a_{1}, \ldots, a_{s}$, not all zero, such that $a_{1} L_{1}+\cdots+a_{s} L_{s}$ vanishes identically on $U$. Otherwise, $L_{1}, \ldots, L_{s}$ are said to be linearly independent on $U$.

Our main tool is the so-called Parametric Subspace Theorem which is stated in Proposition 7.1 below. We consider symmetric convex bodies

$$
\Pi(H):=\left\{\mathbf{x} \in \mathbf{R}^{n}:\left|L_{i}(\mathbf{x})\right| \leq H^{-c_{i}}(i=1, \ldots, r)\right\}
$$

where $r \geq n, L_{1}, \ldots, L_{r}$ are linear forms with real algebraic coefficients in the $n$ variables $X_{1}, \ldots, X_{n}, c_{1}, \ldots, c_{r}$ are reals, and $H$ is a real $\geq 1$. We will refer to $c_{i}$ as the $H$-exponent corresponding to $L_{i}$.

Proposition 7.1. Assume that there are indices $i_{1}, \ldots, i_{n} \in\{1, \ldots, r\}$ such that

$$
\operatorname{rank}\left(L_{i_{1}}, \ldots, L_{i_{n}}\right)=n, c_{i_{1}}+\cdots+c_{i_{n}}>0 .
$$

Then there is a finite collection of proper linear subspaces $\left\{T_{1}, \ldots, T_{t}\right\}$ of $\mathbf{Q}^{n}$ such that for every $H \geq 1$ there is $T_{i} \in\left\{T_{1}, \ldots, T_{t}\right\}$ with

$$
\Pi(H) \cap \mathbf{Z}^{n} \subset T_{i} .
$$


Proof. This is a special case of [11, Theorem 1.1], where a quantitative version was given with an explicit upper bound for the number of subspaces $t$. In fact, in its qualitative form this result was already proved implicitly by Schmidt.

Lemma 7.2. Let $L_{1}, \ldots, L_{r}$ be linear forms in $X_{1}, \ldots, X_{n}$ with real algebraic coefficients and with $\operatorname{rank}\left(L_{1}, \ldots, L_{r}\right)=n$, let $c_{1}, \ldots, c_{r}$ be reals, and let $\left\{M_{1}, \ldots, M_{s}\right\}$ be a (possibly empty) collection of linear forms in $X_{1}, \ldots, X_{n}$ with complex coefficients. Assume that for every non-zero linear subspace $U$ of $\mathbf{Q}^{n}$ on which none of $M_{1}, \ldots, M_{s}$ vanishes identically there are indices $i_{1}, \ldots, i_{m} \in$ $\{1, \ldots, r\}(m=\operatorname{dim} U)$ such that

$$
L_{i_{1}}, \ldots, L_{i_{m}} \text { are linearly independent on } U, \quad c_{i_{1}}+\cdots+c_{i_{m}}>0 \text {. }
$$

Then there is $H_{0}>1$ such that if there is $\mathbf{x}$ with

$$
\mathbf{x} \in \Pi(H) \cap \mathbf{Z}^{n}, \mathbf{x} \neq 0, M_{j}(\mathbf{x}) \neq 0 \text { for } j=1, \ldots, s,
$$

then $H \leq H_{0}$.

Proof. Denote by $\Lambda(H)$ the set of points $\mathbf{x} \in \Pi(H) \cap \mathbf{Z}^{n}$ with $\mathbf{x} \neq \mathbf{0}$ and $M_{j}(\mathbf{x}) \neq 0$ for $j=1, \ldots, s$. We first prove by decreasing induction on $m(n \geq m \geq 1)$ that there is a finite collection $\mathcal{U}_{m}$ of $m$-dimensional linear subspaces of $\mathbf{Q}^{n}$ such that for every $H \geq 1$ there is a subspace $U \in \mathcal{U}_{m}$ with

$$
\Lambda(H) \subset U
$$

For $m=n$ this is of course obvious. Suppose our assertion has been proved for some integer $m$ with $n \geq m \geq 2$. We proceed to prove it for $m-1$ instead of $m$. Take $U$ from the collection $\mathcal{U}_{m}$, and consider those $H \geq 1$ for which $\Lambda(H)$ is non-empty and contained in $U$. Assuming that such $H$ exist, it follows that none of $M_{1}, \ldots, M_{s}$ vanishes identically on $U$. By a suitable linear transformation we can bijectively map $U$ to $\mathbf{Q}^{m}, U \cap \mathbf{Z}^{n}$ to $\mathbf{Z}^{m}$ and $\Pi(H) \cap \mathbf{R} U$ to a convex body similar to (7.1) of dimension $m$. Our hypothesis (7.3) implies that this convex body satisfies the analogue of condition (7.2) in Proposition 7.1. By applying Proposition 7.1 and then mapping back to $U$, we infer that there is a finite collection $\mathcal{V}_{U}$ of $(m-1)$ dimensional linear subspaces of $U$, such that for every real $H$ under consideration, there is $V \in \mathcal{V}_{U}$ with

$$
\Lambda(H) \subset V .
$$

Now it follows that our assertion holds for $m-1$ instead of $m$, with for $\mathcal{U}_{m-1}$ the union of the collections $\mathcal{V}_{U}$ with $U \in \mathcal{U}_{m}$. This completes our induction step.

By applying the above with $m=1$, we infer that there is a finite collection $\mathcal{U}_{1}=\left\{W_{1}, \ldots, W_{w}\right\}$ of one-dimensional linear subspaces of $\mathbf{Q}^{n}$, such that for every $H \geq 1$ there is $W_{i} \in \mathcal{U}_{1}$ with

$$
\Lambda(H) \subset W_{i} .
$$

Let $W$ be one of the subspaces from $\mathcal{U}_{1}$. Choose a non-zero vector $\mathbf{x}_{0} \in W \cap \mathbf{Z}^{n}$ whose coefficients have gcd 1 . Such a vector is up to sign uniquely determined by 
$W$. Suppose that there exists $H \geq 1$ for which $\Lambda(H)$ is non-empty and contained in $W$. By dividing any point in $\Lambda(H)$ by the gcd of its coordinates we obtain $\mathbf{x}_{0} \in \Lambda(H)$. This implies $M_{j}\left(\mathbf{x}_{0}\right) \neq 0$ for $j=1, \ldots s$, and so by assumption (7.3), there is $i \in\{1, \ldots, r\}$ such that $L_{i}\left(\mathbf{x}_{0}\right) \neq 0$ and $c_{i}>0$. Further,

$$
\left|L_{i}\left(\mathbf{x}_{0}\right)\right| \leq H^{-c_{i}} .
$$

Hence $H \leq H_{W}$ for some finite constant $H_{W}$ depending only on $W$.

Now Lemma 7.2 is satisfied with $H_{0}=\max _{i=1, \ldots, w} H_{W_{i}}$.

Denote by $\lambda_{1}(H), \ldots, \lambda_{n}(H)$ the successive minima of $\Pi(H)$. Recall that $\lambda_{i}(H)$ is the minimum of all positive reals $\lambda$ such that $\lambda \Pi(H)$ contains $i$ linearly independent points from $\mathbf{Z}^{n}$.

Lemma 7.3. Let $L_{1}, \ldots, L_{r}$ be linear forms in $X_{1}, \ldots, X_{n}$ with real algebraic coefficients and with $\operatorname{rank}\left(L_{1}, \ldots, L_{r}\right)=n$ and let $c_{1}, \ldots, c_{r}$ be reals. Put

$$
E:=\frac{1}{n} \max \left\{c_{i_{1}}+\cdots+c_{i_{n}}\right\}
$$

where the maximum is taken over all tuples $i_{1}, \ldots, i_{n}$ such that $L_{i_{1}}, \ldots, L_{i_{n}}$ are linearly independent.

(i) There is a constant $c>0$ depending only on $n, L_{1}, \ldots, L_{r}$ such that for every $H \geq 1$ we have $\lambda_{1}(H) \leq c H^{E}$.

(ii) Assume that for every non-zero linear subspace $U$ of $\mathbf{Q}^{n}$ there are indices $i_{1}, \ldots, i_{m} \in\{1, \ldots, r\}(m=\operatorname{dim} U)$ such that

$$
L_{i_{1}}, \ldots, L_{i_{m}} \text { are linearly independent on } U, \quad \frac{1}{m}\left(c_{i_{1}}+\cdots+c_{i_{m}}\right) \geq E \text {. }
$$

Then for every $\varepsilon>0$ there is $H_{\varepsilon}>1$ such that for every $H>H_{\varepsilon}$ we have

$$
H^{E-\varepsilon}<\lambda_{1}(H) \leq \cdots \leq \lambda_{n}(H)<H^{E+\varepsilon} .
$$

Proof. In what follows, the constants implied by $\ll$ and $\gg$ may depend on $L_{1}, \ldots, L_{r}$, $c_{1}, \ldots, c_{r}, n, \varepsilon$, but are independent of $H$. Without loss of generality, $L_{1}, \ldots, L_{n}$ are linearly independent and $c_{1} \geq \cdots \geq c_{r}$.

We first prove (i). Let $\Pi^{\prime}(H)$ be the set of $\mathbf{x} \in \mathbf{R}^{n}$ with $\left|L_{i}(\mathbf{x})\right| \leq H^{-c_{i}}$ for $i=1, \ldots, n$ (so with only $n$ instead of $r$ inequalities). There is a constant $\lambda_{0}>0$ such that $\Pi(H) \supseteq \lambda_{0} \Pi^{\prime}(H)$ and this implies at once

$$
\operatorname{Vol}(\Pi(H)) \gg \operatorname{Vol}\left(\Pi^{\prime}(H)\right) \gg H^{-\left(c_{1}+\cdots+c_{n}\right)}=H^{-n E} .
$$

So by Minkowski's Theorem on successive minima,

$$
\prod_{i=1}^{n} \lambda_{i}(H) \ll H^{n E}
$$

This implies (i). 
We now prove (ii), and assume that for every non-zero linear subspace $U$ of $\mathbf{Q}^{n}$ there are indices $i_{1}, \ldots, i_{m}$ with (7.5). Let $\varepsilon>0$. We first show that for every sufficiently large $H$ we have

$$
\lambda_{1}(H)>H^{E-\varepsilon / n},
$$

in other words, that for every sufficiently large $H$ the convex body

$$
H^{E-\varepsilon / n} \Pi(H)=\left\{\mathbf{x} \in \mathbf{R}^{n}:\left|L_{i}(\mathbf{x})\right| \leq H^{E-c_{i}-\varepsilon / n}(i=1, \ldots, r)\right\}
$$

does not contain non-zero points $\mathbf{x}$ in $\mathbf{Z}^{n}$.

We apply Lemma 7.2 with $c_{i}-E+\varepsilon / n$ instead of $c_{i}$ for $i=1, \ldots, r$. From our assumption it follows that for every non-zero linear subspace $U$ of $\mathbf{Q}^{n}$ there are indices $i_{1}, \ldots, i_{m}(m=\operatorname{dim} U)$ such that $L_{i_{1}}, \ldots, L_{i_{m}}$ are linearly independent on $U$ and

$$
\sum_{j=1}^{m}\left(c_{i_{j}}-E+\varepsilon / n\right)=\left(\sum_{j=1}^{m} c_{i_{j}}\right)-m E+m \varepsilon / n>0 .
$$

So condition (7.3) is satisfied, and therefore we have $H^{E-\varepsilon / n} \Pi(H) \cap \mathbf{Z}^{n}=\{\mathbf{0}\}$ for every sufficiently large $H$. This proves (7.7).

Now a combination of (7.7) with (7.6) immediately gives (ii).

Let $n$ be a positive integer and $\xi$ a complex, non-real algebraic number of degree larger than $n$. Define the linear forms

$$
L_{1}:=\operatorname{Re}\left(\sum_{i=0}^{n} \xi^{i} X_{i}\right), \quad L_{2}:=\operatorname{Im}\left(\sum_{i=0}^{n} \xi^{i} X_{i}\right),
$$

and the symmetric convex body

$$
\begin{gathered}
K(\xi, n, w, H):=\left\{\mathbf{x} \in \mathbf{R}^{n+1}:\left|L_{1}(\mathbf{x})\right| \leq H^{-w},\left|L_{2}(\mathbf{x})\right| \leq H^{-w},\right. \\
\left.\left|x_{0}\right| \leq H, \ldots,\left|x_{n}\right| \leq H\right\},
\end{gathered}
$$

where $\mathbf{x}=\left(x_{0}, \ldots, x_{n}\right)$ and $w \in \mathbf{R}$. We denote by $\lambda_{i}(\xi, n, w, H)(i=1, \ldots, n+1)$ the successive minima of this body.

Recall that $V_{n}(\mu, \xi)$ consists of the polynomials $f \in \mathbf{Q}[X]$ of degree at most $n$ for which $\mu f(\xi) \in \mathbf{R}$. We start with a simple lemma.

\section{Lemma 7.4.}

(i) Let $U$ be a non-zero linear subspace of $\mathbf{Q}^{n+1}$. Then at least one of the linear forms $L_{1}, L_{2}$ does not vanish identically on $U$.

(ii) Let $U$ be a linear subspace of $\mathbf{Q}^{n+1}$. Then $L_{1}, L_{2}$ are linearly dependent on $U$ if and only if there is $\mu \in \mathbf{C}^{*}$ such that

$$
U \subset\left\{\mathbf{x} \in \mathbf{Q}^{n+1}: \sum_{i=0}^{n} x_{i} X^{i} \in V_{n}(\mu, \xi)\right\} .
$$


Proof.

(i) If $L_{1}, L_{2}$ would both vanish identically on $U$, then so would $L_{1}+\sqrt{-1} \cdot L_{2}=$ $\sum_{i=0}^{n} x_{i} \xi^{i}$. But this is impossible since $\xi$ has degree larger than $n$.

(ii) The linear forms $L_{1}, L_{2}$ are linearly dependent on $U$ if and only if there are $\alpha, \beta \in \mathbf{R}$ such that $\alpha L_{1}+\beta L_{2}$ is identically zero on $U$. Using

$$
\alpha L_{1}(\mathbf{x})+\beta L_{2}(\mathbf{x})=\operatorname{Im}\left(\mu \sum_{i=0}^{n} x_{i} \xi^{i}\right) \text { with } \mu=\beta+\sqrt{-1} \cdot \alpha
$$

one verifies at once that $L_{1}, L_{2}$ are linearly dependent on $U$ if and only if for every $\mathbf{x} \in U$ the polynomial $\sum_{i=0}^{n} x_{i} X^{i}$ belongs to $V_{n}(\mu, \xi)$.

Let $t_{n}(\xi)$ be the quantity defined by (2.5). By Lemma 4.2, we have either $t_{n}(\xi) \leq$ $\frac{n+1}{2}$ or $t_{n}(\xi)=\frac{n+2}{2}$. In what follows we have to distinguish between these two cases. In the proofs below, constants implied by $\ll$ and $\gg$ may depend on $\xi, n, w$, and on an additional parameter $\varepsilon$, but are independent of $H$.

Lemma 7.5. Assume that $t_{n}(\xi) \leq(n+1) / 2$ and let $w \geq-1$.

(i) There is a constant $c=c(\xi, n)>0$ such that for every $H \geq 1$ we have $\lambda_{1}(\xi, n, w, H) \leq c H^{\frac{2 w-n+1}{n+1}}$.

(ii) For every $\varepsilon>0$ there is $H_{1, \varepsilon}>1$ such that for every $H>H_{1, \varepsilon}$ we have

$$
H^{\frac{2 w-n+1}{n+1}-\varepsilon}<\lambda_{1}(\xi, n, w, H) \leq \cdots \leq \lambda_{n+1}(\xi, n, w, H)<H^{\frac{2 w-n+1}{n+1}+\varepsilon} .
$$

Proof. In the situation being considered here, for the quantity $E$ defined by (7.4) we have $E=\frac{2 w-n+1}{n+1}$. Thus, part (i) of Lemma 7.5 follows at once from part (i) of Lemma 7.3.

We deduce part (ii) of Lemma 7.5 from part (ii) of Lemma 7.3. and to this end we have to verify the conditions of the latter. First let $U$ be a linear subspace of $\mathbf{Q}^{n+1}$ of dimension $m>t_{n}(\xi)$. By part (ii) of Lemma 7.4, the linear forms $L_{1}, L_{2}$ are linearly independent on $U$. Pick $m-2$ linear forms from $X_{0}, \ldots, X_{n}$ which together with $L_{1}, L_{2}$ are linearly independent on $U$. Then the sum of the $H$-exponents corresponding to these linear forms is equal to $2 w-m+2$, and

$$
\frac{2 w-m+2}{m} \geq \frac{2 w-n+1}{n+1}=E .
$$

Now let $U$ be a non-zero linear subspace of $\mathbf{Q}^{n+1}$ of dimension $m \leq t_{n}(\xi)$. By part (i) of Lemma 7.4, there is a linear form $L_{i} \in\left\{L_{1}, L_{2}\right\}$ which does not vanish identically on $U$. Pick $m-1$ linear forms from $X_{0}, \ldots, X_{n}$ which together with $L_{i}$ are linearly independent on $U$. Then the sum of the $H$-exponents corresponding to these linear forms is $w-m+1$, and again

$$
\frac{w-m+1}{m} \geq \frac{w-\frac{1}{2}(n+1)+1}{\frac{1}{2}(n+1)}=E
$$


where we have used $m \leq t_{n}(\xi) \leq \frac{n+1}{2}$. Hence, indeed, the conditions of part (ii) of Lemma 7.3 are satisfied. This proves part (ii) of Lemma 7.5.

We now deal with the case that $t_{n}(\xi)=\frac{n+2}{2}$. Choose $\mu_{0} \in \mathbf{C}^{*}$ such that $\operatorname{dim} V_{n}\left(\mu_{0}, \xi\right)=t_{n}(\xi)$ and define

$$
\begin{aligned}
U_{0}: & =\left\{\mathbf{x} \in \mathbf{Q}^{n+1}: \sum_{i=0}^{n} x_{i} X^{i} \in V_{n}\left(\mu_{0}, \xi\right)\right\} \\
& =\left\{\mathbf{x} \in \mathbf{Q}^{n+1}: \mu_{0} \sum_{i=0}^{n} x_{i} \xi^{i} \in \mathbf{R}\right\} .
\end{aligned}
$$

Then $\operatorname{dim} U_{0}=t_{n}(\xi)$ and by Lemma 4.1 the vector space $U_{0}$ does not depend on the choice of $\mu_{0}$. Recall that we can choose $\mu_{0}$ from $\mathbf{Q}(\xi)$. Thus, $\mu_{0}$ is algebraic.

Lemma 7.6. Assume that $t_{n}(\xi)=\frac{n+2}{2}$ and let $w \geq-1$.

(i) There is a constant $c=c(\xi, n)>0$ such that for every $H \geq 1$ we have $\lambda_{1}(\xi, n, w, H) \leq c H^{\frac{2 w-n}{n+2}}$.

(ii) For every $\varepsilon>0$ there is $H_{2, \varepsilon}>0$ such that for every $H>H_{2, \varepsilon}$ we have

$$
\begin{aligned}
& H^{\frac{2 w-n}{n+2}-\varepsilon}<\lambda_{1}(\xi, n, w, H) \leq \cdots \leq \lambda_{(n+2) / 2}(\xi, n, w, H)<H^{\frac{2 w-n}{n+2}+\varepsilon} . \\
& H^{\frac{2 w-n+2}{n}-\varepsilon}<\lambda_{(n+4) / 2}(\xi, n, w, H) \leq \cdots \leq \lambda_{n+1}(\xi, n, w, H)<H^{\frac{2 w-n+2}{n}+\varepsilon} . \\
& H^{\frac{2 w-n+2}{n}-\varepsilon} K(\xi, n, w, H) \cap \mathbf{Z}^{n+1} \subset U_{0} .
\end{aligned}
$$

Proof. We first prove part (ii). The idea is to apply Lemma 7.3 first to a convex body defined on the quotient space $\mathbf{R}^{n+1} / \mathbf{R} U_{0}$, and then to $K(\xi, n, w, H)$ restricted to $\mathbf{R} U_{0}$.

Let $\mu_{0}=\alpha_{0}+\sqrt{-1} \cdot \beta_{0}$, where $\alpha_{0}, \beta_{0} \in \mathbf{R}$ and define the linear form

$$
M_{1}:=\frac{1}{\left|\alpha_{0}\right|+\left|\beta_{0}\right|} \cdot\left(\beta_{0} L_{1}+\alpha_{0} L_{2}\right) .
$$

By a straightforward computation,

$$
M_{1}=\frac{1}{2 \sqrt{-1}\left(\left|\alpha_{0}\right|+\left|\beta_{0}\right|\right)}\left(\mu_{0} \sum_{i=0}^{n} \xi^{i} X_{i}-\overline{\mu_{0}} \sum_{i=0}^{n} \bar{\xi}^{i} X_{i}\right),
$$

hence

$$
\left\{\mathbf{x} \in \mathbf{Q}^{n+1}: M_{1}(\mathbf{x})=0\right\}=U_{0} .
$$

Since $U_{0}$ has dimension $\frac{n+2}{2}$, we can choose linear forms $M_{2}, \ldots, M_{n / 2}$ in $X_{0}, \ldots, X_{n}$ as follows: $M_{2}, \ldots, M_{n / 2}$ vanish identically on $U_{0} ;\left\{M_{1}, M_{2}, \ldots, M_{n / 2}\right\}$ 
is linearly independent; and each $M_{i}\left(i=2, \ldots, \frac{n}{2}\right)$ has real algebraic coefficients the sum of whose absolute values is equal to 1 .

There is a surjective linear map $\psi$ from $\mathbf{R}^{n+1}$ to $\mathbf{R}^{n / 2}$ with kernel $\mathbf{R} U_{0}$, which induces a surjective $\mathbf{Z}$-linear map from $\mathbf{Z}^{n+1}$ to $\mathbf{Z}^{n / 2}$ with kernel $U_{0} \cap \mathbf{Z}^{n+1}$. For $i=1, \ldots, \frac{n}{2}$, let $M_{i}^{*}$ be the linear form on $\mathbf{R}^{n / 2}$ such that $M_{i}=M_{i}^{*} \circ \psi$. Then $M_{1}^{*}, \ldots, M_{n / 2}^{*}$ are linearly independent. Now it is clear that for $\mathbf{x} \in K(\xi, n, w, H)$ we have

$$
\begin{aligned}
& \left|M_{1}^{*}(\psi(\mathbf{x}))\right|=\left|M_{1}(\mathbf{x})\right| \leq \max \left(\left|L_{1}(\mathbf{x})\right|,\left|L_{2}(\mathbf{x})\right|\right) \leq H^{-w}, \\
& \left|M_{i}^{*}(\psi(\mathbf{x}))\right|=\left|M_{i}(\mathbf{x})\right| \leq \max \left(\left|x_{0}\right|, \ldots,\left|x_{n}\right|\right) \leq H \quad(i=2, \ldots, n / 2),
\end{aligned}
$$

in other words, if $\mathbf{x} \in K(\xi, n, w, H)$ then $\psi(\mathbf{x})$ belongs to the convex body

$$
\Pi(H):=\left\{\mathbf{y} \in \mathbf{R}^{n / 2}:\left|M_{1}^{*}(\mathbf{y})\right| \leq H^{-w},\left|M_{i}^{*}(\mathbf{y})\right| \leq H(i=2, \ldots, n / 2)\right\} .
$$

Similarly, for any $\lambda>0$ we have

$$
\mathbf{x} \in \lambda K(\xi, n, w, H) \cap \mathbf{Z}^{n+1} \Longrightarrow \psi(\mathbf{x}) \in \lambda \Pi(H) \cap \mathbf{Z}^{n / 2} .
$$

Let $\varepsilon>0$. Denote by $v_{1}(H), \ldots, v_{n / 2}(H)$ the successive minima of $\Pi(H)$. We apply Lemma 7.3. Let $U$ be a linear subspace of $\mathbf{Q}^{n / 2}$ of dimension $m>0$. By (7.15), $M_{1}^{*}$ does not vanish identically on $U$. Pick $m-1$ linear forms from $M_{2}^{*}, \ldots M_{n / 2}^{*}$ which together with $M_{1}^{*}$ form a system of linear forms linearly independent on $U$. The sum of the $H$-exponents corresponding to these linear forms is $w-m+1$ and we have

$$
\frac{w-m+1}{m} \geq \frac{2 w-n+2}{n} .
$$

So the conditions of part (ii) of Lemma 7.3 are satisfied. Consequently, for every sufficiently large $H$ we have

$$
H^{\frac{2 w-n+2}{n}-\varepsilon / 2 n}<v_{1}(H) \leq \cdots \leq v_{n / 2}(H)<H^{\frac{2 w-n+2}{n}+\varepsilon / 2 n} .
$$

Together with (7.16) this implies

$$
H^{\frac{2 w-n+2}{n}-\varepsilon / 2 n} K(\xi, n, w, H) \cap \mathbf{Z}^{n+1} \subset U_{0}
$$

which implies (7.14).

Further, since $\operatorname{dim} U_{0}=\frac{n}{2}+1$, we have

$$
H^{\frac{2 w-n+2}{n}-(\varepsilon / 2 n)}<\lambda_{\frac{n+4}{2}}(\xi, n, w, H) \leq \cdots \leq \lambda_{n+1}(\xi, n, w, H) .
$$

For $i=1, \ldots, \frac{n+2}{2}$, denote by $\mu_{i}(H)$ the minimum of all positive reals $\mu$ such that $\mu K(\xi, n, w, H) \cap U_{0} \cap \mathbf{Z}^{n+1}$ contains $i$ linearly independent points. 
We apply again Lemma 7.3. Let $U$ be a linear subspace of $U_{0}$ of dimension $m>0$. By part (i) of Lemma 7.4, there is a linear form $L_{i} \in\left\{L_{1}, L_{2}\right\}$ which does not vanish identically on $U$. Pick $m-1$ coordinates from $x_{0}, \ldots, x_{n}$ which together with $L_{i}$ form a system of linear forms which is linearly independent on $U$. Then the sum of the $H$-exponents corresponding to these linear forms is $w-m+1$ and

$$
\frac{w-m+1}{m} \geq \frac{2 w-n}{n+2} .
$$

By means of a bijective linear map $\phi$ from $\mathbf{R} U_{0}$ to $\mathbf{R}^{(n+2) / 2}$ with $\phi\left(U_{0} \cap \mathbf{Z}^{n+1}\right)=$ $\mathbf{Z}^{(n+2) / 2}$, we can transform $K(\xi, n, w, H) \cap \mathbf{R} U_{0}$ into a convex body with successive minima $\mu_{1}(H), \ldots, \mu_{(n+2) / 2}(H)$ satisfying the conditions of part (ii) of Lemma 7.3. It follows that for every sufficiently large $H$,

$$
H^{\frac{2 w-n}{n+2}-\varepsilon / 2 n}<\mu_{1}(H) \leq \cdots \leq \mu_{\frac{n+2}{2}}(H)<H^{\frac{2 w-n}{n+2}+\varepsilon / 2 n} .
$$

By combining (7.18) with (7.17) and the already proved (7.14) we obtain (assuming that $\varepsilon$ is sufficiently small), that $\mu_{i}(H)=\lambda_{i}(\xi, n, w, H)$ for $i=1, \ldots, \frac{n+2}{2}$. By inserting this into (7.18) we obtain (7.12).

By Minkowski's theorem,

$$
\prod_{i=1}^{n+1} \lambda_{i}(\xi, n, w, H) \ll \operatorname{Vol}(K(\xi, n, w, H))^{-1} \ll H^{\frac{2 w-n+1}{n+1}} .
$$

Together with (7.12), (7.17) this implies that for every sufficiently large $H$ we have

$$
H^{\frac{2 w-n+2}{n}-\varepsilon / 2 n}<\lambda_{\frac{n+4}{2}}(\xi, n, w, H) \leq \cdots \leq \lambda_{n+1}(\xi, n, w, H)<H^{\frac{2 w-n+2}{n}+\varepsilon} .
$$

This implies (7.13), and completes the proof of part (ii).

It remains to prove part (i). Applying part (i) of Lemma 7.3 to the image under $\phi$ of $K(\xi, n, w, H) \cap \mathbf{R} U_{0}$ we obtain that there is a constant $c=c(\xi, n)>0$ such that for every $H \geq 1$ we have $\mu_{1}(H) \leq H^{\frac{2 w-n}{n+2}}$. Since obviously, $\lambda_{1}(\xi, n, w, H) \leq$ $\mu_{1}(H)$, part (i) follows.

\section{Proof of Theorem 2.5}

Let again $n$ be a positive integer, and $\xi$ a complex, non-real algebraic number of degree $>n$. Let $L_{1}, L_{2}$ denote the linear forms defined by (7.8) and $K(\xi, n, w, H)$ the convex body defined by (7.9). Put

$$
u_{n}(\xi):=\max \left\{\frac{n-1}{2}, t_{n}(\xi)-1\right\} .
$$

In view of Lemma 4.4, in order to prove Theorem 2.5, it suffices to prove that $w_{n}(\xi) \leq u_{n}(\xi), \hat{w}_{n}(\xi) \geq u_{n}(\xi), w_{n}^{*}(\xi) \geq u_{n}(\xi)$. 
Lemma 8.1. We have $w_{n}(\xi) \leq u_{n}(\xi)$.

Proof. Let $w \in \mathbf{R}$. Suppose there are infinitely many polynomials $P=x_{0}+x_{1} X+$ $\cdots+x_{n} X^{n} \in \mathbf{Z}[X]$ satisfying

$$
0<|P(\xi)| \leq H(P)^{-w} .
$$

For such a polynomial $P$, put $H:=H(P), \mathbf{x}=\left(x_{0}, \ldots, x_{n}\right)$. Then clearly, $\left|L_{1}(\mathbf{x})\right|=|\operatorname{Re} P(\xi)| \leq H^{-w},\left|L_{2}(\mathbf{x})\right|=|\operatorname{Im} P(\xi)| \leq H^{-w},\left|x_{i}\right| \leq H$ for $i=0, \ldots, n$, and so

$$
\mathbf{x} \in K(\xi, n, w, H) \cap \mathbf{Z}^{n+1} .
$$

Since (8.2) is supposed to hold for infinitely many polynomials $P \in \mathbf{Z}[X]$ of degree $\leq n$, there are arbitrarily large $H$ such that there is a non-zero $\mathbf{x}$ with (8.3). That is, there are arbitrarily large $H$ such that the first minimum $\lambda_{1}=\lambda_{1}(\xi, n, w, H)$ of $K(\xi, n, w, H)$ is $\leq 1$.

First suppose that $t_{n}(\xi) \leq \frac{n+1}{2}$. Then $u_{n}(\xi)=\frac{n-1}{2}$. By Lemma 7.5, for every $\varepsilon>0$ there is $H_{\varepsilon}>1$ such that $\lambda_{1} \geq H^{\frac{2 w-n+1}{n+1}-\varepsilon}$ for every $H>H_{\varepsilon}$. Hence $w \leq \frac{n-1}{2}=u_{n}(\xi)$. Now suppose that $t_{n}(\xi)=\frac{n+2}{2}$; then $u_{n}(\xi)=\frac{n}{2}$. By Lemma 7.6, for every $\varepsilon>0$ there is $H_{\varepsilon}>1$ such that $\lambda_{1} \geq H^{\frac{2 w-n}{n+2}-\varepsilon}$ for every $H>H_{\varepsilon}$. Hence $w \leq \frac{n}{2}=u_{n}(\xi)$. This implies Lemma 8.1.

Lemma 8.2. We have $\hat{w}_{n}(\xi) \geq u_{n}(\xi), w_{n}^{*}(\xi) \geq u_{n}(\xi)$.

Proof. We prove the following stronger assertion: For every $\varepsilon>0$ there is $H_{\varepsilon}>1$ such that for every $H>H_{\varepsilon}$ there is a non-zero irreducible polynomial $P \in \mathbf{Z}[X]$ of degree $n$ with

$$
0<|P(\xi)| \leq H^{-u_{n}(\xi)+\varepsilon}, \quad\left|P^{\prime}(\xi)\right| \geq H^{1-\varepsilon}, \quad H(P) \leq H,
$$

where $P^{\prime}$ denotes the derivative of $P$.

By ignoring the lower bound for $\left|P^{\prime}(\xi)\right|$ in (8.4) we obtain that for every $H>$ $H_{\varepsilon}$ there is a non-zero irreducible polynomial $P \in \mathbf{Z}[X]$ of degree $n$ such that $0<|P(\xi)| \leq H^{-u_{n}(\xi)+\varepsilon}$. This implies $\hat{w}_{n}(\xi) \geq u_{n}(\xi)$.

To prove that $w_{n}^{*}(\xi) \geq u_{n}(\xi)$ we have to show that for every $\varepsilon>0$ there are infinitely many algebraic numbers $\alpha$ of degree at most $n$ with $|\xi-\alpha| \leq H(\alpha)^{-u_{n}(\xi)-1+\varepsilon}$. We prove the existence of infinitely many such $\alpha$ of degree equal to $n$. Take an irreducible polynomial $P \in \mathbf{Z}[X]$ with (8.4) and let $\alpha$ be a zero of $P$ closest to $\xi$. Then using the inequalities $|\xi-\alpha| \ll\left|P(\xi) / P^{\prime}(\xi)\right|$ (see [2, (A.11) on page 228]) and $H(\alpha) \ll H(P) \ll H$, we obtain

$$
|\xi-\alpha| \ll H^{-u_{n}(\xi)-1+2 \varepsilon} \ll H(\alpha)^{-u_{n}(\xi)-1+2 \varepsilon},
$$

where the constants implied by $\ll$ depend only on $n, \varepsilon$. Since deg $\xi>n$, the number $\alpha$ cannot be equal to $\xi$ so equation (8.5) cannot hold for fixed $\alpha$ and arbitrarily 
large $H$. Hence by letting $H \rightarrow \infty$, we obtain infinitely many distinct algebraic numbers $\alpha$ of degree $n$ with (8.5).

We proceed to prove the assertion stated above. Constants implied by $\ll$ and $\gg$ will depend on $\xi, n$ and $\varepsilon$. Write the polynomial $P$ as $P=x_{0}+x_{1} X+\cdots+x_{n} X^{n}$ and put $\mathbf{x}:=\left(x_{0}, \ldots, x_{n}\right)$. As before, let $L_{1}, L_{2}$ be the linear forms given by $L_{1}(\mathbf{x})=\operatorname{Re} P(\xi), L_{2}(\mathbf{x})=\operatorname{Im} P(\xi)$. Further, define the linear forms $L_{1}^{\prime}, L_{2}^{\prime}$ by

$$
\begin{aligned}
& L_{1}^{\prime}(\mathbf{x})=\operatorname{Re} P^{\prime}(\xi)=\operatorname{Re}\left(\sum_{j=1}^{n} j x_{j} \xi^{j-1}\right), \\
& L_{2}^{\prime}(\mathbf{x})=\operatorname{Im} P^{\prime}(\xi)=\operatorname{Im}\left(\sum_{j=1}^{n} j x_{j} \xi^{j-1}\right) .
\end{aligned}
$$

We have to distinguish between the cases $t_{n}(\xi) \leq \frac{n+1}{2}$ and $t_{n}(\xi)=\frac{n+2}{2}$.

First suppose that $t_{n}(\xi) \leq \frac{n+1}{2}$. Then $u_{n}(\xi)=\frac{n-1}{2}$. We prove that for every $\varepsilon>0$ there is $H_{\varepsilon}>1$ with the property that for every $H>H_{\varepsilon}$ there is $\mathbf{x} \in \mathbf{Z}^{n+1}$ with

$$
\begin{aligned}
& \left|L_{1}(\mathbf{x})\right| \leq H^{-\frac{n-1}{2}+\varepsilon / 3},\left|L_{2}(\mathbf{x})\right| \leq H^{-\frac{n-1}{2}+\varepsilon / 3}, \quad\left|x_{0}\right| \leq H, \ldots,\left|x_{n}\right| \leq H, \\
& \max \left\{\left|L_{1}^{\prime}(\mathbf{x})\right|,\left|L_{2}^{\prime}(\mathbf{x})\right|\right\}>H^{1-\varepsilon}, \\
& 2 \not x_{n}, \quad 2 \mid x_{i} \text { for } i=0, \ldots, n-1,4 \not x_{0} .
\end{aligned}
$$

Then the polynomial $P=\sum_{i=0}^{n} x_{i} X^{i}$ satisfies (8.4) and by Eisenstein's criterion it is irreducible.

Let $H \geq 1, \varepsilon>0$. Consider vectors $\mathbf{x} \in \mathbf{Z}^{n+1}$ satisfying (8.6) but not (8.7), i.e., with

$$
\left\{\begin{array}{l}
\left|L_{1}(\mathbf{x})\right| \leq H^{-\frac{n-1}{2}+\varepsilon / 3},\left|L_{2}(\mathbf{x})\right| \leq H^{-\frac{n-1}{2}+\varepsilon / 3}, \\
\left|L_{1}^{\prime}(\mathbf{x})\right| \leq H^{1-\varepsilon},\left|L_{2}^{\prime}(\mathbf{x})\right| \leq H^{1-\varepsilon}, \\
\left|x_{0}\right| \leq H, \ldots,\left|x_{n}\right| \leq H .
\end{array}\right.
$$

By considering the coefficients of $X_{0}, X_{1}, X_{2}$ one infers that the linear forms $L_{1}$, $L_{2}$ and $L_{2}^{\prime}$ are linearly independent. Pick $n-2$ coordinates from $X_{0}, \ldots, X_{n}$ which together with $L_{1}, L_{2}, L_{2}^{\prime}$ form a system of $n+1$ linearly independent linear forms. The sum of the corresponding $H$-exponents is

$$
(n-1-2 \varepsilon / 3)+(\varepsilon-1)-(n-2)=\varepsilon / 3>0 .
$$

So by Proposition 7.1, there is a finite collection of proper linear subspaces $T_{1}, \ldots, T_{m}$ of $\mathbf{Q}^{n+1}$, with the property that for every $H \geq 1$, there is $T_{i} \in\left\{T_{1}, \ldots, T_{m}\right\}$ such that the set of solutions $\mathbf{x} \in \mathbf{Z}^{n+1}$ of (8.9) is contained in $T_{i}$. Consequently, if $\mathbf{x}$ satisfies (8.6) but does not lie in $T_{1} \cup \ldots \cup T_{m}$ then it also satisfies (8.7).

We apply Lemma 7.5 with $w=\frac{n-1}{2}$. Let $\eta>0$ be a parameter depending on $n, \varepsilon$ to be chosen later, and $Y$ a parameter depending on $H$ and $\eta$, also chosen 
later. For brevity we write $K(Y)$ for the convex body $K\left(\xi, n, \frac{n-1}{2}, Y\right)$ and denote by $\lambda_{n+1}(Y)$ the largest of the successive minima of this body. According to a result of Mahler (see Cassels [6, Lemma 8, page 135]) there is a constant $c_{1}=c_{1}(n)$ such that the convex body $c_{1} \lambda_{n+1}(Y) K(Y)$ contains a basis of $\mathbf{Z}^{n+1}$. By applying Lemma 5.2 with $\frac{\eta}{2}$ instead of $\varepsilon$ we obtain that for every sufficiently large $Y$ we have $\lambda_{n+1}(Y)<Y^{\eta / 2}$. Then for every $Y$ large enough to satisfy also $c_{1} Y^{\eta / 2}<Y^{\eta}$, the convex body $Y^{\eta} K(Y)$, that is, the body given by

$$
\left|L_{1}(\mathbf{x})\right| \leq Y^{-\frac{n-1}{2}+\eta},\left|L_{2}(\mathbf{x})\right| \leq Y^{-\frac{n-1}{2}+\eta}, \quad\left|x_{0}\right| \leq Y^{1+\eta}, \ldots,\left|x_{n}\right| \leq Y^{1+\eta}
$$

contains a basis of $\mathbf{Z}^{n+1},\left\{\mathbf{x}^{(0)}, \ldots, \mathbf{x}^{(n)}\right\}$, say. Consider the vectors

$$
\mathbf{x}=\left(x_{0}, \ldots, x_{n}\right)=\sum_{i=0}^{n} a_{i} \mathbf{x}^{(i)} \text { with } a_{i} \in \mathbf{Z},\left|a_{i}\right| \leq Y^{\eta} \text { for } i=0, \ldots, n .
$$

Assuming again that $Y$ is sufficiently large, each vector (8.10) satisfies

$$
\left|L_{1}(\mathbf{x})\right| \leq Y^{-\frac{n-1}{2}+3 \eta},\left|L_{2}(\mathbf{x})\right| \leq Y^{-\frac{n-1}{2}+3 \eta},\left|x_{0}\right| \leq Y^{1+3 \eta}, \ldots,\left|x_{n}\right| \leq Y^{1+3 \eta} .
$$

Since $\mathbf{x}^{(0)}, \ldots, \mathbf{x}^{(n)}$ span $\mathbf{Z}^{n+1}$, the number of vectors (8.10) with the additional property $(8.8)$ is $\gg Y^{(n+1) \eta}$. On the other hand, the number of vectors (8.10) lying in $T_{1} \cup \cdots \cup T_{m}$ is $\ll Y^{n \eta}$. Hence if $Y$ is sufficiently large, there exist vectors $\mathbf{x}$ with (8.10), (8.8) and with $\mathbf{x} \notin T_{1} \cup \cdots \cup T_{m}$. Now by choosing $\eta$ and then $Y$ such that

$$
\frac{\frac{n-1}{2}-3 \eta}{1+3 \eta}=\frac{n-1}{2}-\frac{\varepsilon}{3}, \quad Y^{1+3 \eta}=H,
$$

system (8.11) translates into (8.6). Thus, we infer that for every sufficiently large $H$, there exist vectors $\mathbf{x} \in \mathbf{Z}^{n+1}$ with (8.6), (8.8) which do not lie in $T_{1} \cup \cdots \cup T_{m}$. But as we have seen above, such vectors satisfy (8.7). This settles the case that $t_{n}(\xi) \leq \frac{n+1}{2}$.

Now assume that $t_{n}(\xi)=\frac{n+2}{2}$. Then $u_{n}(\xi)=\frac{n}{2}$. We first show that it suffices to prove that for every $\varepsilon>0$ and every sufficiently large $H$ there exists a polynomial $P \in \mathbf{Z}[X]$ of degree $\leq n$ with (8.4), without the requirements that $P$ be irreducible and have degree equal to $n$. Indeed suppose that for every sufficiently large $H$ there is a polynomial $P \in \mathbf{Z}[X]$ satisfying (8.4) such that $\operatorname{deg} P<n$ or $P$ is reducible. By the same argument as above, it follows that there are infinitely many algebraic numbers $\alpha$ of degree $<n$ with (8.5). Then there is $m<n$ such that (8.5) has infinitely many solutions in algebraic numbers $\alpha$ of degree $m$. By Lemma 4.2 and our assumption $t_{n}(\xi)=\frac{n+2}{2}$, the number $n$ is even, so $n-1$ is odd and hence $t_{n-1}(\xi) \leq \frac{n}{2}$. So $u_{n-1}(\xi)=\frac{n-2}{2}<u_{n}(\xi)$. Now by Lemmata 4.4 and 8.1,

$$
w_{m}^{*}(\xi) \leq w_{m}(\xi) \leq w_{n-1}(\xi)<u_{n}(\xi),
$$


which contradicts that (8.5) has infinitely many solutions in algebraic numbers $\alpha$ of degree $m$. So for every sufficiently large $H$, the polynomials $P \in \mathbf{Z}[X]$ of degree $\leq n$ that satisfy (8.4) necessarily have degree equal to $n$ and are irreducible.

Let $\varepsilon>0$. Let $U_{0}$ be the vector space defined by (7.11). Recall that $U_{0}$ has dimension $\frac{n+2}{2}$. We show that for every sufficiently large $H$ there is a non-zero $\mathbf{x} \in U_{0} \cap \mathbf{Z}^{n+1}$ with

$$
\begin{aligned}
& \left|L_{1}(\mathbf{x})\right| \leq H^{-\frac{n}{2}+\varepsilon / 3},\left|L_{2}(\mathbf{x})\right| \leq H^{-\frac{n}{2}+\varepsilon / 3},\left|x_{0}\right| \leq H, \ldots,\left|x_{n}\right| \leq H, \\
& \max \left\{\left|L_{1}^{\prime}(\mathbf{x})\right|,\left|L_{2}^{\prime}(\mathbf{x})\right|\right\}>H^{1-\varepsilon} .
\end{aligned}
$$

Let $H>1$ and consider those vectors $\mathbf{x} \in U_{0} \cap \mathbf{Z}^{n+1}$ satisfying (8.12) but not (8.13), i.e.,

$$
\left\{\begin{array}{l}
\left|L_{1}(\mathbf{x})\right| \leq H^{-\frac{n}{2}+\varepsilon / 3},\left|L_{2}(\mathbf{x})\right| \leq H^{-\frac{n}{2}+\varepsilon / 3} \\
\left|L_{1}^{\prime}(\mathbf{x})\right| \leq H^{1-\varepsilon},\left|L_{2}^{\prime}(\mathbf{x})\right| \leq H^{1-\varepsilon} \\
\left|x_{0}\right| \leq H, \ldots,\left|x_{n}\right| \leq H
\end{array}\right.
$$

Claim. There are $L_{i} \in\left\{L_{1}, L_{2}\right\}, L_{j}^{\prime} \in\left\{L_{1}^{\prime}, L_{2}^{\prime}\right\}$ that are linearly independent on $U_{0}$.

Assume the contrary. By Lemma 7.4, the linear forms $L_{1}, L_{2}$ are linearly dependent on $U_{0}$ and at least one of $L_{1}, L_{2}$ does not vanish identically on $U_{0}$. Hence $M:=L_{1}+\sqrt{-1} \cdot L_{2}$ does not vanish identically on $U_{0}$, and so $M$ and $M^{\prime}:=L_{1}^{\prime}+\sqrt{-1} \cdot L_{2}^{\prime}$ are linearly dependent on $U_{0}$.

Since $\operatorname{dim} U_{0}=\frac{n+2}{2}$, there are two linearly independent vectors $\mathbf{a}=\left(a_{0}, \ldots, a_{n}\right)$, $\mathbf{b}=\left(b_{0}, \ldots, b_{n}\right) \in U_{0} \cap \mathbf{Z}^{n+1}$ such that if $k$ is the largest index with $a_{k} \neq 0$ and $l$ the largest index with $b_{l} \neq 0$, then $k<l \leq n-\frac{n+2}{2}+2=\frac{n+2}{2}$. Let $A=\sum_{i=0}^{k} a_{i} X^{i}$, $B=\sum_{j=0}^{l} b_{j} X^{j}$.

Then by the linear dependence of $M, M^{\prime}$ we have

$$
A(\xi) B^{\prime}(\xi)-A^{\prime}(\xi) B(\xi)=M(\mathbf{a}) M^{\prime}(\mathbf{b})-M^{\prime}(\mathbf{a}) M(\mathbf{b})=0 .
$$

But the polynomial $A B^{\prime}-A^{\prime} B$ is not identically 0 (since $A, B$ are linearly independent) and has degree at most

$$
k+l-1 \leq 2(n+1-(n+2) / 2)=n .
$$

This leads to a contradiction since by assumption, $\operatorname{deg} \xi>n$. This proves our claim.

Choose $\frac{n-2}{2}$ coordinates from $X_{0}, \ldots, X_{n}$ which together with $L_{i}, L_{j}^{\prime}$ form a system of $\frac{n+2}{2}$ linear forms which are linearly independent on $U_{0}$. Then the sum of the corresponding $H$-exponents is

$$
\left(\frac{n}{2}-\varepsilon / 3\right)+(-1+\varepsilon)-\frac{n-2}{2}=2 \varepsilon / 3>0 .
$$


So by Proposition 7.1, there are proper linear subspaces $T_{1}, \ldots, T_{m}$ of $U_{0}$ with the property that for every $H>1$ there is $T_{i} \in\left\{T_{1}, \ldots, T_{m}\right\}$ such that the set of $\mathbf{x} \in U_{0} \cap \mathbf{Z}^{n+1}$ with (8.14) is contained in $T_{i}$. This implies that vectors $\mathbf{x} \in U_{0} \cap \mathbf{Z}^{n+1}$ that satisfy (8.12) and for which $\mathbf{x} \notin T_{1} \cap \cdots \cap T_{m}$ necessarily have to satisfy (8.13).

To show that there are vectors $\mathbf{x} \in U_{0} \cap \mathbf{Z}^{n+1}$ with $\mathbf{x} \notin T_{1} \cup \cdots \cup T_{m}$ one proceeds similarly as above, but applying Lemma 7.6 with $w=\frac{n}{2}$ instead of Lemma 7.5: For appropriate $\eta, Y$, depending on $\varepsilon, H$, one may choose a basis $\mathbf{x}^{(1)}, \ldots, \mathbf{x}^{\left(\frac{n+2}{2}\right)}$ of $U_{0} \cap \mathbf{Z}^{n+1}$ in $c_{2} \lambda_{(n+2) / 2}(Y) K(Y)$, where $c_{2}=c_{2}(n)$ depends only on $n$, and $\lambda_{(n+2) / 2}(Y)$ is the $\frac{n+2}{2}$-th minimum of $K(Y):=K\left(\xi, n, \frac{n}{2}, Y\right)$. Then one takes linear combinations as in (8.10), and by a counting argument arrives at a vector $\mathbf{x}$ with (8.12) which does not lie in $T_{1} \cup \cdots \cup T_{m}$, hence satisfies (8.13). Here, we don't have to impose (8.8). This completes the proof of Lemma 8.2.

\section{Proof of Theorem 3.1}

We first prove the following analogue of Theorem 2.5.

Proposition 9.1. Let $n$ be a positive integer and $\xi$ a complex, non-real algebraic number of degree $>n$. Then

$$
\widetilde{w}_{n}(\xi)=\widetilde{w}_{n}^{*}(\xi)=\hat{\widetilde{w}}_{n}(\xi)=n-1-\max \left\{\frac{n-1}{2}, t_{n}(\xi)-1\right\} .
$$

Put $v_{n}(\xi):=n-1-\max \left\{\frac{n-1}{2}, t_{n}(\xi)-1\right\}$. Completely similarly as in Lemma 4.4 we have

$$
\widetilde{w}_{n}^{*}(\xi) \leq \widetilde{w}_{n}(\xi), \quad \hat{\widetilde{w}}_{n}(\xi) \leq \widetilde{w}_{n}(\xi)
$$

Therefore, in order to prove Proposition 9.1, it suffices to prove the inequalities

$$
\widetilde{w}_{n}^{*}(\xi) \geq v_{n}(\xi), \quad \hat{\widetilde{w}}_{n}(\xi) \geq v_{n}(\xi), \quad \widetilde{w}_{n}(\xi) \leq v_{n}(\xi) .
$$

These inequalities are proved in Lemmata 9.2 and 9.3 below. The integer $n$ and the algebraic number $\xi$ will be as in the statement of Proposition 9.1.

Lemma 9.2. We have

$$
\widetilde{w}_{n}^{*}(\xi) \geq v_{n}(\xi), \quad \hat{\widetilde{w}}_{n}(\xi) \geq v_{n}(\xi)
$$

Proof. We proceed as in Bugeaud and Teulié [5], using a method developed by Davenport and Schmidt [9] (see also [2, Theorem 2.11]). As in Section 7, we consider the symmetric convex body

$$
\begin{aligned}
K(\xi, n, w, H):=\left\{\mathbf{x} \in \mathbf{R}^{n+1}:\left|x_{n} \operatorname{Re}\left(\xi^{n}\right)+\ldots+x_{1} \operatorname{Re}(\xi)+x_{0}\right|\right. & \leq H^{-w}, \\
\left|x_{n} \operatorname{Im}\left(\xi^{n}\right)+\ldots+x_{1} \operatorname{Im}(\xi)+x_{0}\right| & \leq H^{-w}, \\
\left|x_{0}\right| \leq H, \ldots,\left|x_{n}\right| & \leq H\},
\end{aligned}
$$

where $\mathbf{x}=\left(x_{0}, \ldots, x_{n}\right)$ and $w \in \mathbf{R}$. 
Set $w:=v_{n}(\xi)$. For brevity, we denote the convex body $K(\xi, n, w, H)$ by $K(H)$.

Let $\varepsilon>0$ be a real number. Then in both the cases $t_{n}(\xi) \leq(n+1) / 2$ and $t_{n}(\xi)=(n+2) / 2$ we have, by Lemmata 7.5 and 7.6, respectively, that for every sufficiently large real number $H$,

$$
\lambda_{n+1}(H)<H^{\varepsilon},
$$

where $\lambda_{n+1}(H)$ denotes the largest successive mimimum of $K(H)$.

There is a constant $c_{1}=c_{1}(n)$ such that the convex body $c_{1} \lambda_{n+1}(H) K(H)$ contains a basis of $\mathbf{Z}^{n+1}$,

$$
\mathbf{x}^{(i)}=\left(x_{0}^{(i)}, \ldots, x_{n}^{(i)}\right)(i=1, \ldots, n+1),
$$

say. This means that there exist $n+1$ integer polynomials

$$
P_{i}=x_{n}^{(i)} X^{n}+\ldots+x_{1}^{(i)} X+x_{0}^{(i)}, \quad(i=1, \ldots, n+1),
$$

that form a basis of the $\mathbf{Z}$-module of polynomials in $\mathbf{Z}[X]$ of degree at most $n$ and for which

$$
H\left(P_{i}\right) \leq c_{1} H^{1+\varepsilon}, \quad(1 \leq i \leq n+1),
$$

and

$$
\max \left\{\left|\operatorname{Re}\left(P_{i}(\xi)\right)\right|,\left|\operatorname{Im}\left(P_{i}(\xi)\right)\right|\right\} \leq c_{1} H^{-w+\varepsilon}, \quad(1 \leq i \leq n+1) .
$$

There is a unique polynomial $Q=X^{n+1}+\sum_{i=0}^{n} y_{i} X^{i} \in \mathbf{R}[X]$ such that

$$
\left\{\begin{array}{l}
\operatorname{Re} Q(\xi)=H^{-w+2 \varepsilon}, \quad \operatorname{Im} Q(\xi)=H^{-w+2 \varepsilon}, \quad \operatorname{Im} Q^{\prime}(\xi)=H^{1+2 \varepsilon}, \\
y_{3}=\cdots=y_{n}=0 .
\end{array}\right.
$$

Indeed, if we express $\operatorname{Re} Q(\xi), \operatorname{Im} Q(\xi)$ and $\operatorname{Im} Q^{\prime}(\xi)$ as linear forms in $y_{0}, \ldots, y_{n}$ they form together with $y_{3}, \ldots, y_{n}$ a linearly independent system of rank $n+1$, and so (9.3) gives rise to a system of linear equations with a unique solution $y_{0}, \ldots, y_{n}$. tain

By expressing $y_{0}, y_{1}, y_{2}$ as a linear combination of these linear forms, we ob-

$$
\left|y_{i}\right| \ll H^{1+2 \varepsilon} \text { for } i=0,1,2,
$$

where here and below, constants implied by $\ll$ depend on $n, \xi$, $\varepsilon$ only. Since $P_{1}, \ldots, P_{n+1}$ span the vector space of polynomials with real coefficients of degree at most $n$, there are reals $\theta_{1}, \ldots, \theta_{n+1}$ such that

$$
Q=X^{n+1}+2 \sum_{i=1}^{n+1} \theta_{i} P_{i}
$$


Now choose integers $t_{1}, \ldots, t_{n}$ with

$$
\left|\theta_{i}-t_{i}\right| \leq 1 \quad(i=1, \ldots, n+1),
$$

and define the polynomial

$$
P:=X^{n+1}+2 \sum_{i=1}^{n+1} t_{i} P_{i} .
$$

Write $P=X^{n+1}+\sum_{i=1}^{n} x_{i} X^{i}$.

For a suitable choice of $t_{1}, \ldots, t_{n+1}$, the polynomial $P$ is irreducible. Indeed, since $P_{1}, \ldots, P_{n+1}$ span the $\mathbf{Z}$-module of all integer polynomials of degree at most $n$, at least one of the constant terms $x_{0}^{(1)}, \ldots, x_{0}^{(n+1)}$ of $P_{1}, \ldots, P_{n+1}$, respectively, must be odd. Without loss of generality we assume this to be $x_{0}^{(1)}$. For a fixed $n$-tuple $\left(t_{2}, \ldots, t_{n+1}\right)$, there are two choices for $t_{1}$, that we denote by $t_{1,0}$ and $t_{1,1}=t_{1,0}+1$. Since $x_{0}^{(1)}$ is odd, we can choose $t_{1} \in\left\{t_{1,0}, t_{1,1}\right\}$ such that $t_{1} x_{0}^{(1)}+\cdots+t_{n+1} x_{0}^{(n+1)}$ is odd. Then the constant coefficient of $P$, namely $2\left(t_{1} x_{0}^{(1)}+\ldots+t_{n+1} x_{0}^{(n+1)}\right)$, is not divisible by 4 , and the irreducibility of $P$ follows from the Eisenstein criterion applied with the prime number 2.

From (9.5), (9.1), it follows that the absolute values of the coefficients of $P-Q$ are $\ll H^{1+\varepsilon}$. Further, by (9.2), (9.1) we have

$$
\begin{aligned}
& |\operatorname{Re} P(\xi)-\operatorname{Re} Q(\xi)| \ll H^{-w+\varepsilon}, \quad|\operatorname{Im} P(\xi)-\operatorname{Im} Q(\xi)| \ll H^{-w+\varepsilon}, \\
& \left|\operatorname{Im} P^{\prime}(\xi)-\operatorname{Im} Q^{\prime}(\xi)\right| \ll H^{1+\varepsilon} .
\end{aligned}
$$

Together with (9.3), (9.4) this implies, assuming that $H$ is sufficiently large,

$$
H(P) \leq H^{1+3 \varepsilon},
$$

and moreover,

$$
|P(\xi)| \leq|\operatorname{Re} P(\xi)|+|\operatorname{Im} P(\xi)| \leq H^{-w+3 \varepsilon}, \quad\left|P^{\prime}(\xi)\right| \geq\left|\operatorname{Im} P^{\prime}(\xi)\right| \geq H^{1+\varepsilon} .
$$

Ignoring the lower bound for $\left|P^{\prime}(\xi)\right|$, we infer that

$$
\hat{\widetilde{w}}_{n}(\xi) \geq(w-3 \varepsilon) /(1+3 \varepsilon) .
$$

Since $\varepsilon$ is arbitrary, we get the second statement of the lemma. Furthermore, we deduce that the monic polynomial $P$ has a complex root $\alpha$ with

$$
|\xi-\alpha| \ll \frac{|P(\xi)|}{\left|P^{\prime}(\xi)\right|} \ll H(\alpha)^{-(w+1-2 \varepsilon) /(1+3 \varepsilon)} .
$$

Since $\varepsilon$ is arbitrary, this shows that

$$
\widetilde{w}_{n}^{*}(\xi) \geq w=v_{n}(\xi),
$$

and the proof of Lemma 9.2 is complete.

We now prove an upper bound for $\widetilde{w}_{n}(\xi)$. 
Lemma 9.3. We have

$$
\widetilde{w}_{n}(\xi) \leq v_{n}(\xi)
$$

Proof. It suffices to show that for every $w>v_{n}(\xi)$, the inequality

$$
0<|P(\xi)| \leq H(P)^{-w}
$$

has only finitely many solutions in monic polynomials $P \in \mathbf{Z}[X]$ of degree at most $n+1$. By replacing any monic polynomial $P$ of degree $k<n+1$ satisfying (9.8) by $X^{n-k} P$ and modifying $w$ a little bit, one easily observes that it suffices to show that for every $w>v_{n}(\xi)$, inequality (9.8) has only finitely many solutions in monic polynomials $P \in \mathbf{Z}[X]$ of degree precisely $n+1$.

We have again to distinguish between the cases $t_{n}(\xi) \leq \frac{n+1}{2}$ and $t_{n}(\xi)=\frac{n+2}{2}$. The first case is dealt with by a modification of the proof of Lemma 7.5, and the second by a modification of the proof of Lemma 7.6.

First assume that $t_{n}(\xi) \leq \frac{n+1}{2}$. Then $v_{n}(\xi)=\frac{n-1}{2}$. Consider the inequality (9.8) to be solved in monic polynomials $P \in \mathbf{Z}[X]$ of degree $n+1$. Define the polynomial $P=\sum_{i=0}^{n+1} x_{i} X^{i}$ where $x_{n+1}=1$ and put $\mathbf{x}=\left(x_{0}, \ldots, x_{n}, x_{n+1}\right)$, $H:=H(P)$. Define the linear forms

$$
\tilde{L}_{1}:=\operatorname{Re}\left(\sum_{i=0}^{n+1} \xi^{i} X_{i}\right), \quad \tilde{L}_{2}:=\operatorname{Im}\left(\sum_{i=0}^{n+1} \xi^{i} X_{i}\right), \quad \tilde{M}:=\sum_{i=0}^{n+1} \xi^{i} X_{i} .
$$

Then we can translate (9.8) into

$$
\left\{\begin{array}{l}
\left|\tilde{L}_{1}(\mathbf{x})\right| \leq H^{-w},\left|\tilde{L}_{2}(\mathbf{x})\right| \leq H^{-w} \\
\left|x_{0}\right| \leq H, \ldots,\left|x_{n}\right| \leq H,\left|x_{n+1}\right| \leq 1, x_{n+1} \neq 0, \tilde{M}(\mathbf{x}) \neq 0 .
\end{array}\right.
$$

We prove that for every $w>\frac{n-1}{2}$ there is $H_{w}>1$ such that if (9.9) has a solution $\mathbf{x} \in \mathbf{Z}^{n+2}$ then $H<H_{w}$. This implies at once that for every $w>\frac{n-1}{2}$ there are only finitely many monic polynomials $P \in \mathbf{Z}[X]$ of degree $\leq n+1$ with (9.8), and hence that $\widetilde{w}_{n}(\xi) \leq \frac{n-1}{2}=v_{n}(\xi)$.

We apply Lemma 7.2. Let $w>\frac{n-1}{2}$. We have to verify (7.3). First, let $U$ be a linear subspace of $\mathbf{Q}^{n+2}$ of dimension $m>\frac{n+3}{2}$ on which $X_{n+1}$ and $\tilde{M}$ are not identically 0 . Then $\tilde{L}_{1}, \tilde{L}_{2}, X_{n+1}$ are linearly independent on $U$. For if not, then the linear forms

$$
L_{1}:=\operatorname{Re}\left(\sum_{i=0}^{n} \xi^{i} X_{i}\right), L_{2}:=\operatorname{Im}\left(\sum_{i=0}^{n} \xi^{i} X_{i}\right)
$$


are linearly dependent on $U \cap\left\{x_{n+1}=0\right\}$ which has dimension larger than $\frac{n+1}{2}$. But by part (ii) of Lemma 7.4 this is impossible. Now choose $m-3$ coordinates from $X_{0}, \ldots, X_{n}$ which together with $\tilde{L}_{1}, \tilde{L}_{2}, X_{n+1}$ are linearly independent on $U$. Then the $H$-exponents corresponding to these linear forms have sum

$$
2 w+0+(3-m)>n+2-m \geq 0 .
$$

Now let $U$ be a linear subspace of $\mathbf{Q}^{n+2}$ of dimension $m$ with $2 \leq m \leq \frac{n+3}{2}$ on which $X_{n+1}, \tilde{M}$ do not vanish identically. Then there is $\tilde{L}_{i} \in\left\{\tilde{L}_{1}, \tilde{L}_{2}\right\}$ such that $\tilde{L}_{i}$ and $X_{n+1}$ are linearly independent on $U$. For if not then both $L_{1}$ and $L_{2}$ vanish identically on $U \cap\left\{x_{n+1}=0\right\}$ which is impossible by part (i) of Lemma 7.4. Choose $m-2$ coordinates from $X_{0}, \ldots, X_{n}$ which together with $\tilde{L}_{i}$ and $X_{n+1}$ are linearly independent on $U$. Then the $H$-exponents corresponding to these linear forms have sum

$$
w+0+(2-m)>\frac{n-1}{2}+2-m \geq 0 .
$$

Finally, let $U$ be a one-dimensional linear subspace of $\mathbf{Q}^{n+2}$ on which none of $X_{n+1}, \tilde{M}$, vanishes identically. Then there is $\tilde{L}_{i} \in\left\{\tilde{L}_{1}, \tilde{L}_{2}\right\}$ not vanishing identically on $U$, and the $H$-exponent corresponding to this linear form is $w>0$. We conclude that condition (7.3) of Lemma 7.2 is satisfied. So indeed there is $H_{w}>0$ such that if (9.9) is satisfied by some $\mathbf{x} \in \mathbf{Z}^{n+1}$ then $H<H_{w}$. This settles the case that $t_{n}(\xi) \leq \frac{n+1}{2}$.

Now assume that $t_{n}(\xi)=\frac{n+2}{2}$. Then $v_{n}(\xi)=\frac{n-2}{2}$. Further, by Lemmata 4.2 and 4.3, $n$ is even, $n+1<\operatorname{deg} \xi$, and

$$
t_{n+1}(\xi)=t_{n}(\xi)=\frac{n+2}{2} .
$$

Choose $\mu_{0}=\alpha_{0}+\sqrt{-1} \cdot \beta_{0}$ with $\alpha_{0}, \beta_{0} \in \mathbf{R}$ such that $\operatorname{dim} V_{n}\left(\mu_{0}, \xi\right)=t_{n}(\xi)=$ $\frac{n+2}{2}$. Define the linear form

$$
\begin{aligned}
\tilde{M}_{1} & =\frac{1}{\left|\alpha_{0}\right|+\left|\beta_{0}\right|} \cdot\left(\beta_{0} \tilde{L}_{1}+\alpha_{0} \tilde{L}_{2}\right) \\
& =\frac{1}{2 \sqrt{-1}\left(\left|\alpha_{0}\right|+\left|\beta_{0}\right|\right)}\left(\mu_{0} \sum_{i=0}^{n+1} x_{i} \xi^{i}-\overline{\mu_{0}} \sum_{i=0}^{n+1} x_{i} \bar{\xi}^{i}\right) .
\end{aligned}
$$

Let

$$
\tilde{U}_{0}=\left\{\mathbf{x} \in \mathbf{Q}^{n+2}: \tilde{M}_{1}(\mathbf{x})=0\right\} .
$$

Then $\mathbf{x}=\left(x_{0}, \ldots, x_{n+1}\right) \in \tilde{U}_{0}$ if and only if $\sum_{i=0}^{n+1} x_{i} X^{i} \in V_{n+1}\left(\mu_{0}, \xi\right)$. 
We claim that $X_{n+1}=0$ identically on $\tilde{U}_{0}$. Suppose $\tilde{U}_{0}$ contains a vector $\mathbf{x}=\left(x_{0}, \ldots, x_{n+1}\right)$ with $x_{n+1} \neq 0$. Then the polynomial $\sum_{i=0}^{n+1} x_{i} X^{i}$ belongs to $V_{n+1}\left(\mu_{0}, \xi\right)$ but not to $V_{n}\left(\mu_{0}, \xi\right)$ which is impossible by (9.10). This argument shows also that $\operatorname{dim} \tilde{U}_{0}=\operatorname{dim} V_{n}\left(\mu_{0}, \xi\right)=\frac{n+2}{2}$.

There are linear forms $\tilde{M}_{2}, \ldots, \tilde{M}_{n / 2}$ in $X_{0}, \ldots, X_{n+1}$ with the following properties: $\tilde{M}_{2}, \ldots, \tilde{M}_{n / 2}$ vanish indentically on $\tilde{U}_{0} ;\left\{\tilde{M}_{1}, \tilde{M}_{2}, \ldots, \tilde{M}_{n / 2}, X_{n+1}\right\}$ is linearly independent; and each $\tilde{M}_{i}\left(i=2, \ldots, \frac{n}{2}\right)$ has real algebraic coefficients whose absolute values have sum equal to 1 .

Let $\psi$ be a surjective linear mapping from $\mathbf{R}^{n+2}$ to $\mathbf{R}^{\frac{n+2}{2}}$ with kernel $\mathbf{R} \tilde{U}_{0}$ such that the restriction of $\psi$ to $\mathbf{Z}^{n+2}$ maps surjectively to $\mathbf{Z}^{\frac{n+2}{2}}$ and has kernel $\tilde{U}_{0} \cap \mathbf{Z}^{n+2}$. For $i=1, \ldots \frac{n}{2}$, let $\tilde{M}_{i}^{*}$ be the linear form on $\mathbf{R}^{\frac{n+2}{2}}$ with $\tilde{M}_{i}=\tilde{M}_{i}^{*} \circ \psi$. Further, let $\tilde{M}_{0}^{*}$ be the linear form on $\mathbf{R}^{\frac{n+2}{2}}$ such that $X_{n+1}=\tilde{M}_{0}^{*} \circ \psi$. Then $\tilde{M}_{0}^{*}, \ldots, \tilde{M}_{n / 2}^{*}$ are linearly independent.

Let $w>v_{n}(\xi)=\frac{n-2}{2}$. Let $P \in \mathbf{Z}[X]$ be a monic polynomial of degree $n+1$ satisfying (9.8). Write $P=\sum_{i=0}^{n+1} x_{i} X^{i}, x_{n+1}=1, \mathbf{x}=\left(x_{0}, \ldots, x_{n+1}\right), H:=$ $H(P)$. Then $\mathbf{x}$ satisfies (9.9). By an easy computation it follows that $\mathbf{y}:=\psi(\mathbf{x})$ satisfies

$$
\left\{\begin{array}{l}
\left|\tilde{M}_{1}^{*}(\mathbf{y})\right| \leq H^{-w},\left|\tilde{M}_{i}^{*}(\mathbf{y})\right| \leq H(i=2, \ldots, n / 2), \\
\left|\tilde{M}_{0}^{*}(\mathbf{y})\right| \leq 1, M_{0}^{*}(\mathbf{y}) \neq 0 .
\end{array}\right.
$$

We show that system (9.11) satisfies condition (7.3) of Lemma 7.2. First let $U=$ $\mathbf{Q}^{\frac{n+2}{2}}$. As observed before, the linear forms $\tilde{M}_{0}^{*}, \ldots, \tilde{M}_{n / 2}^{*}$ are linearly independent, and the $H$-exponents corresponding to these linear forms have sum

$$
w-(n-(n / 2)-1)+0>0 .
$$

Now let $U$ be a linear subspace of $\mathbf{Q}^{\frac{n+2}{2}}$ of dimension $m$ with $0<m \leq \frac{n}{2}$ on which $\tilde{M}_{0}^{*}$ does not vanish identically. The linear form $\tilde{M}_{1}$ can not vanish identically on $\psi^{-1}(U)$ since $\psi^{-1}(U)$ is strictly larger than $U_{0}$, therefore, $\tilde{M}_{1}^{*}$ does not vanish identically on $U$. Choose $m-1$ linear forms among $\tilde{M}_{0}^{*}, \tilde{M}_{2}^{*}, \ldots, \tilde{M}_{n / 2}^{*}$ which together with $\tilde{M}_{1}^{*}$ are linearly independent on $U$. Then the sum of the $H$-exponents corresponding to these linear forms is at least

$$
w-(m-1) \geq w-((n / 2)-1)>0 .
$$

Hence condition (7.3) of Lemma 7.2 is satisfied. It follows that there is $H_{w}>0$ such that if system (9.11) is solvable in $\mathbf{y} \in \mathbf{Z}^{\frac{n+2}{2}}$ then $H \leq H_{w}$. Hence for every monic polynomial $P \in \mathbf{Z}[X]$ of degree $n+1$ with (9.8) we have $H(P) \leq H_{w}$, implying that (9.8) has only finitely many solutions.

As observed above, Proposition 9.1 follows from Lemmata 9.2 and 9.3. 
Proof of Theorem 3.1. We first prove (3.1). Assume that deg $\xi=: d \leq n+1$. By Liouville's inequality (4.1) we have $\widetilde{w}_{n}(\xi) \leq \frac{d-2}{2}$. By Proposition 9.1 and Lemma 4.3 we have $\tilde{w}_{d-1}^{*}(\xi)=\hat{\widetilde{w}}_{d-1}(\xi)=\frac{d-2}{2}$. Hence

$$
\widetilde{w}_{n}^{*}(\xi) \geq \widetilde{w}_{d-1}^{*}(\xi)=\frac{d-2}{2}, \quad \hat{\widetilde{w}}_{n}(\xi) \geq \hat{\widetilde{w}}_{d-1}(\xi)=\frac{d-2}{2}
$$

These facts together imply (3.1).

The equalities (3.2) and (3.3) follow at once by combining Proposition 9.1 with part (ii) of Lemma 4.2. The last assertion of Theorem 3.1 follows at once from Theorem 2.5 and Proposition 9.1. This completes the proof of Theorem 3.1.

\section{A refined question}

The exponents $w_{n}, \hat{w}_{n}, \ldots$ are defined as suprema of certain sets of real numbers. We may further ask whether the suprema are also maxima. In other words, for a given complex number $\xi$, a positive integer $n$, do there exist a constant $c(\xi, n)$ and infinitely many integer polynomials $P(H)$ of degree at most $n$ such that

$$
0<|P(\xi)| \leq c(\xi, n) H(P)^{-w_{n}(\xi)} ?
$$

This is Problem P.1 on [2, page 210].

When $\xi$ is algebraic and real, the answer is clearly positive, by Dirichlet's theorem. When $\xi$ is algebraic and non-real, we have already seen that $w_{n}(\xi)$ can be much larger than expected; however, the answer to the above question is also positive.

Proposition 10.1. For any positive integer $n$ and any complex, non-real algebraic number $\xi$, there exist a constant $c(\xi, n)>0$ and infinitely many integer polynomials $P(H)$ of degree at most $n$ such that

$$
0<|P(\xi)| \leq c(\xi, n) H(P)^{-w_{n}(\xi)} .
$$

Proof. This follows from (the proof of) Satz 1 from Schmidt [21]; however, we feel that it is better to include a complete proof. Constants implied by $\ll$, 》 depend only on $n, \xi$.

First assume that $d:=\operatorname{deg} \xi>n$. We apply part (i) of Lemmata 7.5 and 7.6, respectively, with $w=w_{n}(\xi)$. Then in view of Theorem 2.5, in both the cases $t_{n}(\xi) \leq \frac{n+1}{2}, t_{n}(\xi)=\frac{n+2}{2}$, we have that for every $H \geq 1$ the first minimum $\lambda_{1}(\xi, n, w, H)$ of the convex body $K(\xi, n, w, H)$ defined by (7.9) is $\ll 1$. Consequently, for every $H \geq 1$, there is a non-zero polynomial $P=\sum_{i=0}^{n} x_{i} X^{i} \in \mathbf{Z}[X]$ such that

$$
\begin{aligned}
& |\operatorname{Re} P(\xi)|=\left|L_{1}(\mathbf{x})\right| \ll H^{-w},|\operatorname{Im} P(\xi)|=\left|L_{2}(\mathbf{x})\right| \ll H^{-w}, \\
& H(P)=\max \left\{\left|x_{0}\right|, \ldots,\left|x_{n}\right|\right\} \ll H .
\end{aligned}
$$


This clearly implies $|P(\xi)| \ll H^{-w} \ll H(P)^{-w}$. Arbitrarily large $H$ cannot give rise to the same polynomial $P$ since otherwise we would have $P(\xi)=0$, against our assumption that $\operatorname{deg} \xi>n$. This proves Proposition 10.1 in the case that $d>n$.

To treat the case $n \geq d$ we simply have to observe that by Theorem 2.1 we have $w_{n}(\xi)=w_{d-1}(\xi)=\frac{d-2}{2}$ and that by what we have proved above, (10.1) has already infinitely many solutions in polynomials $P$ of degree at most $d-1$.

Actually, the above proof yields that the analogue of Proposition 10.1 is true for the uniform exponent of approximation $\hat{w}_{n}$. However, it is a very interesting, but presumably very difficult, question to decide whether the analogue of Proposition 10.1 holds for the exponent $w_{n}^{*}$.

We briefly summarize what is known on this question.

Proposition 10.2. For any positive integer $n$ and any complex algebraic number $\xi$ of degree $n+1$, there exist a constant $c(\xi)$ and infinitely many algebraic numbers $\alpha$ of degree at most $n$ such that

$$
0<|\xi-\alpha| \leq c(\xi) H(\alpha)^{-w_{n}^{*}(\xi)-1} .
$$

Proof. When $\xi$ is real, Proposition 10.2 has been established by Wirsing [25] (see also [2, Theorem 2.9], which reproduces an alternative proof, due to Bombieri and Mueller [1]). Without any additional complication, the same method gives the required result when $\xi$ is complex and non-real.

Furthermore, Davenport and Schmidt [7] proved that for every real algebraic number $\xi$ of degree at least 3, there exist a constant $c(\xi)$ and infinitely many algebraic integer $\alpha$ of degree at most 2 such that

$$
0<|\xi-\alpha| \leq c(\xi) H(\alpha)^{-w_{2}^{*}(\xi)-1}=c(\xi) H(\alpha)^{-3} .
$$

This is a consequence of a more general result of theirs on linear forms $[8,10]$, which is the key tool for the proof of the second assertion of the next proposition.

\section{Proposition 10.3.}

(i) For any complex algebraic number $\xi$ of degree greater than 2, there exist a constant $c(\xi)$ and infinitely many algebraic numbers $\alpha$ of degree at most 2 such that

$$
0<|\xi-\alpha| \leq c(\xi) H(\alpha)^{-w_{2}^{*}(\xi)-1} .
$$

(ii) For any complex algebraic number $\xi$ of degree greater than 4 satisfying $w_{4}^{*}(\xi)=$ 2 , there exist a constant $c(\xi)$ and infinitely many algebraic numbers $\alpha$ of degree at most 4 such that

$$
0<|\xi-\alpha| \leq c(\xi) H(\alpha)^{-w_{4}^{*}(\xi)-1} .
$$


Proof. Let $\xi$ be a complex non-real number of degree greater than 2 . By the proof of Proposition 10.1, there are infinitely many integer quadratic polynomials $P$ satisfying

$$
|P(\xi)| \ll H(P)^{-w_{2}(\xi)}, \quad|P(\bar{\xi})| \ll H(P)^{-w_{2}(\xi)} .
$$

Such a polynomial $P$ has a root very near to $\xi$ and another very near to $\bar{\xi}$. Consequently, it satisfies $\left|P^{\prime}(\xi)\right| \gg H(P)$ and its root $\alpha$ near to $\xi$ is such that $|\xi-\alpha| \ll H(\alpha)^{-w_{2}(\xi)-1}$. This proves the first part of the proposition since $w_{2}(\xi)=w_{2}^{*}(\xi)$.

Let $\xi$ be a complex (non-real) algebraic number of degree $>4$ satisfying $w_{4}^{*}(\xi)=2$. By Theorem 2.5, this means that $t_{4}(\xi)=3$, i.e., there is $\mu_{0}$ such that $\operatorname{dim} V_{4}\left(\mu_{0}, \xi\right)=3$. Let $U_{0}$ be the vector space of $\mathbf{x}=\left(x_{0}, \ldots, x_{4}\right) \in \mathbf{Q}^{5}$ such that $\sum_{i=0}^{4} x_{i} X^{i} \in V_{4}\left(\mu_{0}, \xi\right)$. Define the linear forms $L_{1}, L_{2}, L_{1}^{\prime}, L_{2}^{\prime}$ by

$$
L_{1}(\mathbf{x})=\operatorname{Re} P(\xi), L_{2}(\mathbf{x})=\operatorname{Im} P(\xi), L_{1}^{\prime}(\mathbf{x})=\operatorname{Re} P^{\prime}(\xi), L_{2}^{\prime}(\mathbf{x})=\operatorname{Im} P^{\prime}(\xi)
$$

where $P=\sum_{i=0}^{4} x_{i} X^{i}$. By Lemma 7.4, the linear forms $L_{1}, L_{2}$ are linearly dependent on $U_{0}$. On the other hand, by the claim in the proof of Lemma 8.2, there are $i, j \in\{1,2\}$ such that $L_{i}, L_{j}^{\prime}$ are linearly independent on $U_{0}$. Choose linearly independent polynomials $P_{1}, P_{2}, P_{3}$ from $V_{4}\left(\mu_{0}, \xi\right)$ with integer coefficients. By Lemma 4.2 we may assume $\operatorname{deg} P_{1}<\operatorname{deg} P_{2}<\operatorname{deg} P_{3}=4$. Express $P \in V_{4}\left(\mu_{0}, \xi\right)$ as $y_{1} P_{1}+y_{2} P_{2}+y_{3} P_{3}$ with $\mathbf{y}=\left(y_{1}, y_{2}, y_{3}\right) \in \mathbf{Q}^{3}$. Thus, $\operatorname{Re} P(\xi)$, $\operatorname{Im} P(\xi), \operatorname{Re} P^{\prime}(\xi), \operatorname{Im} P^{\prime}(\xi)$ can be expressed as linear forms in $\mathbf{y}$,

$$
\operatorname{Re} P(\xi)=M_{1}(\mathbf{y}), \operatorname{Im} P(\xi)=M_{2}(\mathbf{y}), \operatorname{Re} P^{\prime}(\xi)=M_{1}^{\prime}(\mathbf{y}), \operatorname{Im} P^{\prime}(\xi)=M_{2}^{\prime}(\mathbf{y})
$$

say, and by the above, $M_{1}, M_{2}$ are linearly dependent and there are $i, j \in\{1,2\}$ such that $M_{i}, M_{j}^{\prime}$ are linearly independent. with

By [8, Theorem 2.1], there are infinitely many integer triples $\mathbf{y}=\left(y_{1}, y_{2}, y_{3}\right)$

$$
\left|M_{i}(\mathbf{y})\right| \ll\left|M_{j}^{\prime}(\mathbf{y})\right| \times\|\mathbf{y}\|^{-3}
$$

where $\|\mathbf{y}\|=\max \left\{\left|y_{1}\right|,\left|y_{2}\right|,\left|y_{3}\right|\right\}$. This implies that there are infinitely many integer polynomials $P$ of degree 4 of the shape $y_{1} P_{1}+y_{2} P_{2}+y_{3} P_{3}$ with $y_{1}, y_{2}, y_{3} \in \mathbf{Z}$ such that

$$
\frac{|P(\xi)|}{\left|P^{\prime}(\xi)\right|} \ll H(P)^{-3} .
$$

Consequently, there are infinitely many algebraic numbers $\alpha$ of degree at most 4 such that $|\xi-\alpha| \ll H(\alpha)^{-3}$. This completes the proof of Proposition 10.3. 


\section{References}

[1] E. BOMBIERI and J. MUeller, Remarks on the approximation to an algebraic number by algebraic numbers, Michigan Math. J. 33 (1986), 83-93.

[2] Y. Bugeaud, "Approximation by Algebraic Numbers", Cambridge Tracts in Mathematics 160, Cambridge University Press, 2004.

[3] Y. Bugeaud and M. Laurent, Exponents of Diophantine approximation and Sturmian continued fractions, Ann. Inst. Fourier (Grenoble) 55 (2005), 773-804.

[4] Y. Bugeaud and M. LaUREnT, On exponents of homogeneous and inhomogeneous Diophantine approximation, Mosc. Math. J. 5 (2005), 747-766.

[5] Y. Bugeaud and O. Teulié, Approximation d'un nombre réel par des nombres algébriques de degré donné, Acta Arith. 93 (2000), 77-86.

[6] J. W. S. CASSELS, "An Introduction to the Geometry of Numbers", Springer Verlag, 1997.

[7] H. DAVENPORT and W. M. SCHMIDT, Approximation to real numbers by quadratic irrationals, Acta Arith. 13 (1967), 169-176.

[8] H. Davenport and W. M. Schmidt, A theorem on linear forms, Acta Arith. 14 (1967/1968), 209-223.

[9] H. DAVENPORT and W. M. SChMidT, Approximation to real numbers by algebraic integers, Acta Arith. 15 (1969), 393-416.

[10] H. DAVENPORT and W. M. SCHMIDT, Dirichlet's theorem on Diophantine approximation II, Acta Arith. 16 (1970), 413-423.

[11] J.-H. EVERTSE and H.P. SCHLICKEWEI, A quantitative version of the Absolute Parametric Subspace Theorem, J. Reine Angew. Math. 548 (2002), 21-127.

[12] A. YA. KhIntChInE, Über eine Klasse linearer diophantischer Approximationen, Rend. Circ. Mat. Palermo 50 (1926), 170-195.

[13] J. F. KокSмa, Über die Mahlersche Klasseneinteilung der transzendenten Zahlen und die Approximation komplexer Zahlen durch algebraische Zahlen, Monatsh. Math. Phys. 48 (1939), 176-189.

[14] K. MAHLER, Zur Approximation der Exponentialfunktionen und des Logarithmus. I, II, J. Reine Angew. Math. 166 (1932), 118-150.

[15] K. F. Roth, Rational approximations to algebraic numbers, Matematika 2 (1955), 1-20; corrigendum, 168.

[16] D. RoY, Approximation simultanée d'un nombre et son carré, C. R. Acad. Sci. Paris 336 (2003), 1-6.

[17] D. Roy, Approximation to real numbers by cubic algebraic numbers, I, Proc. London Math. Soc. 88 (2004), 42-62.

[18] D. Roy, Approximation to real numbers by cubic algebraic numbers, II, Ann. of Math. 158 (2003), 1081-1087.

[19] D. RoY and M. WALDSCHMIDT, Diophantine approximation by conjugate algebraic integers, Compositio Math. 140 (2004), 593-612.

[20] W. M. SchmidT, Simultaneous approximation to algebraic numbers by rationals, Acta Math. 125 (1970), 189-201.

[21] W. M. ScHMIDT, Linearformen mit algebraischen Koeffizienten. II, Math. Ann. 191 (1971), $1-20$.

[22] W. M. SchmidT, "Approximation to Algebraic Numbers", Monographie de l'Enseignement Mathématique 19, Genève, 1971.

[23] W. M. SchmidT, "Diophantine Approximation”, Lecture Notes in Math. 785, Springer, Berlin, 1980.

[24] V. G. SPRINDŽUK, "Mahler's Problem in Metric Number Theory", Izdat. "Nauka i Tehnika", Minsk, 1967 (in Russian). English translation by B. Volkmann, Translations of Mathematical Monographs, Vol. 25, American Mathematical Society, Providence, R.I., 1969. 
[25] E. WIRsING, Approximation mit algebraischen Zahlen beschränkten Grades, J. Reine Angew. Math. 206 (1961), 67-77.

\author{
Université Louis Pasteur \\ U. F. R. de mathématiques \\ 7, rue René Descartes \\ 67084 Strasbourg (France) \\ bugeaud@math.u-strasbg.fr \\ Universiteit Leiden \\ Mathematisch Instituut \\ Postbus 9512 \\ 2300 RA Leiden (The Netherlands) \\ evertse@math.leidenuniv.nl
}

